\title{
The Effect of Moist Convection on the Tropospheric Response to Tropical and Subtropical Zonally Asymmetric Torques
}

\author{
WILLIAM R. BOOS \\ Department of Geology and Geophysics, Yale University, New Haven, Connecticut \\ TIFFANY A. SHAW \\ Department of Earth and Environmental Sciences, and Department of Applied Physics and Applied Mathematics, \\ Columbia University, New York, New York
}

(Manuscript received 5 February 2013, in final form 4 June 2013)

\begin{abstract}
Tropospheric winds can be altered by vertical transfers of momentum due to orographic gravity waves and convection. Previous work showed that, in dry models, such zonally asymmetric torques produce a pattern of tropical ascent that is well described by linear dynamics, together with meridional shifts of the midlatitude jet. Here a series of idealized models is used to understand the effects of moisture on the tropospheric response to tropical and subtropical zonally asymmetric, upper-tropospheric torques.

The vertical motion response to a torque is shown to be amplified by the reduction in effective static stability that occurs in moist convecting atmospheres. This amplification occurs only in precipitating regions, and the magnitude of subsidence in nonprecipitating regions saturates when clear-sky radiative cooling balances induced adiabatic warming. For basic states in which precipitation is concentrated in an intertropical convergence zone (ITCZ), most of the vertical motion response is thus confined within the basic-state ITCZ, even when the torque is remote from the ITCZ. Tropical and subtropical torques perturb the extratropical baroclinic eddy field and the convectively coupled equatorial wave field. Resulting changes in momentum flux convergence by transient eddies induce secondary meridional overturning circulations that modify the zonal-mean response to a torque. The net effect allows tropical torques to merge a double ITCZ into a single equatorial ITCZ. The response of tropical transient eddies is highly sensitive to the representation of convection, so the zonal-mean response to a torque is similarly sensitive, even when the torque is located in the subtropics.
\end{abstract}

\section{Introduction}

Momentum in atmospheres and oceans can be transported by motions with spatial scales orders of magnitude smaller than the planetary radius, yet these momentum transports can alter planetary-scale flow. In Earth's atmosphere, vertical momentum transports are accomplished by orographically excited gravity waves and by the turbulent eddies of convection, processes respectively termed orographic gravity wave drag (OGWD) and convective momentum transport (CMT). Although OGWD and CMT have been the focus of much study and their parameterization in climate models is an active

Corresponding author address: William R. Boos, Department of Geology and Geophysics, Yale University, P.O. Box 208109, New Haven, CT 06520-8109.

E-mail: billboos@alum.mit.edu area of research (e.g., Fritts and Alexander 2003; Richter and Rasch 2008; Stephenson 1994), there is little conceptual understanding of how the associated vertical momentum transports alter the three-dimensional distribution of large-scale tropospheric flow.

To improve our understanding of the influence of such vertical momentum transfers on planetary-scale winds, we explored in previous work the dynamical response to prescribed, zonally confined sources of westward momentum in the tropical and subtropical upper troposphere (Shaw and Boos 2012, hereafter SB12). This choice of forcing was motivated by the qualitative characteristics of vertical momentum flux convergence associated with tropical deep moist convection (e.g., Carr and Bretherton 2001; Lin et al. 2005; Gregory et al. 1997) and orographically excited gravity waves over South Asia (e.g., McFarlane 1987; Palmer et al. 1986). SB12 found that in dry atmospheric models, westward 
torques centered in the tropical upper troposphere produced a roughly linear tropical response consisting of equatorial ascent east of the torque and subsidence in offequatorial anticyclones west of the torque. Just as the linear response to a tropical heating can be explained in terms of the projection of that heating onto equatorially trapped wave modes (e.g., Matsuno 1966; Gill 1980), ascent east of the torque occurs because the forcing projects onto an equatorial Kelvin wave, while off-equatorial subsidence and gyrelike motions west of the torque occur because the forcing projects onto equatorial Rossby waves. Upper-tropospheric equatorward flow in the eastern part of these Rossby gyres advects planetary vorticity that locally balances the vorticity sink associated with the torque. As the upper-tropospheric westward torque was shifted into the subtropics in SB12, subsidence was centered on the polar side of the torque and ascent on the equatorial side, like a zonally confined version of the classic "downward control" response to zonally symmetric midlatitude torques (Haynes et al. 1991). Most westward torques applied in SB12 also produced a poleward shift of the eddy-driven tropospheric jet and a poleward shift of the critical surface for extratropical baroclinic eddies.

While SB12 showed that these responses were robust across linear analytical and nonlinear numerical models, none of those models represented the effects of phase changes of water that are central to the dynamics of Earth's tropical atmosphere. That is the goal of this work: to determine how the tropospheric response to a zonally asymmetric torque is modified by the effects of moisture. All of the torques examined here are prescribed and do not interact with the flow. While this is unrealistic, it is done to build conceptual understanding, just as the linear response to a prescribed tropospheric heating was examined (e.g., Gill 1980) even though the characteristics of that heating will, in the real atmosphere, interact with the flow. It will be shown that the influence of torques on the intertropical convergence zone (ITCZ), the Hadley circulation, and equatorially trapped waves involves sufficient complexity to merit simplification of the problem in an idealized framework.

This is not the first study to examine the influence of torques associated with OGWD and CMT on flow in moist models, but previous studies were limited to comparisons of moist general circulation models (GCMs) integrated with and without parameterized momentum transports. Those studies have shown that parameterizations of unresolved vertical momentum transports can have a large influence on the global distribution of precipitation, the mean meridional circulation, and the position and intensity of extratropical jets (McFarlane 1987; Zhang and McFarlane 1995; Zhou et al. 1996; Wu et al. 2003; Song et al. 2008). However, the complexity of the
GCMs, with time-varying OGWD and CMT and realistic topography and land-ocean contrast, makes it difficult to achieve a deep theoretical understanding of the dynamical response to a torque. This is why Palmer et al. (1986) used an idealized, zonally symmetric dry model to explain results from a GCM, showing how a prescribed, upper-level momentum forcing could alter zonal-mean temperatures and winds by generating a meridional overturning circulation consistent with a downward-controltype balance (Haynes et al. 1991). Furthermore, SB12 showed that a robust, zonally asymmetric dynamical response could be isolated in a linear model and used to understand the nonlinear response. We proceed in a similar spirit here, using idealized models with prescribed, zonally asymmetric torques to better understand the three-dimensional response to OGWD and CMT in a moist convecting atmosphere. Connections with parameterized momentum transfers in GCMs integrated with realistic boundary conditions will be explored in separate, future work.

The next section contains a description of the models used in this study. Results are then presented for basic states with and without meridional temperature gradients, and we conclude with a summary and discussion of implications for the real atmosphere.

\section{Model descriptions}

This study employs two types of numerical models: one with highly simplified representations of both largescale dynamics and subgrid-scale physics, and another based on the primitive equations of motion with detailed parameterizations of subgrid-scale physics. All models use "aquaplanet" boundary conditions lacking any representation of orography or land-ocean contrast.

\section{a. $Q T C M$}

The model with simplified dynamics is version 2.3 of the Quasi-Equilibrium Tropical Circulation Model 1 (QTCM; Neelin and Zeng 2000), which is based on a truncation of the large-scale dynamics to two vertical modes of the tropical troposphere. The simplicity of this model allows the response to a torque to be more readily understood and the effects of various processes to be more easily tested. Most QTCM integrations used a horizontal resolution of $2.5^{\circ}$ latitude $\times 2.8^{\circ}$ longitude with a time step of $600 \mathrm{~s}$. Horizontal diffusivities of $3 \times$ $10^{5} \mathrm{~m}^{2} \mathrm{~s}^{-1}$ were used for temperature and humidity, and a kinematic viscosity of $3.5 \times 10^{5} \mathrm{~m}^{2} \mathrm{~s}^{-1}$ was prescribed. All integrations produced responses that were very nearly in a steady state. Each model configuration was integrated for 400 days with all fields shown here consisting of averages over the last 200 days. A few integrations were 
conducted at the finer resolution of $1.25^{\circ} \times 1.4^{\circ}$ to examine the detailed response in the ITCZ; those integrations used diffusivities of $2.0 \times 10^{5} \mathrm{~m}^{2} \mathrm{~s}^{-1}$ for temperature and humidity, a kinematic viscosity of $2.75 \times 10^{5} \mathrm{~m}^{2} \mathrm{~s}^{-1}$, and a time step of $200 \mathrm{~s}$. Insolation that is uniform in latitude is prescribed, although this affects the atmosphere only through shortwave absorption.

A dry, purely baroclinic version of the QTCM was linearized about a resting state by setting all barotropic wind to zero, setting the convective heat source to zero, and eliminating all nonlinear advective terms in the momentum and temperature equations. Interactive radiation was replaced by Newtonian cooling with the same 13-day time scale used for momentum damping. These changes make the model nearly identical to the linear system solved analytically by SB12, which was obtained by adding a momentum forcing to the system of Gill (1980). Numerical solutions to this linearized QTCM are presented here. Additional QTCM configurations were created by modifying various processes, and relevant details are presented with the model results below.

\section{b. GCMs}

This study also used several versions of the Community Atmosphere Model (CAM; Collins et al. 2006; Neale et al. 2010) of the National Center for Atmospheric Research (NCAR). While our main focus is the moist response to zonally asymmetric torques, we present the response in the same dry model used by SB12 for comparison: version 5.0 (CAM5) with dry idealized physics consisting of Newtonian temperature relaxation and Rayleigh drag in the lower troposphere. This is identical to the Held and Suarez (1994) configuration, but without a meridional gradient in the prescribed equilibrium temperature. This produces a basic state without baroclinic eddies or extratropical jets, allowing for a simpler assessment of the direct response to a prescribed torque.

We also employ two different moist versions of CAM. Most results are from the superparameterized CAM (SP-CAM) version 3.5.1-a GCM in which the moist convection parameterizations have been replaced by a cloud-system resolving model (CSRM) run within each grid cell of the global model (Khairoutdinov and Randall 2001). Each CSRM uses 28 vertical levels and 32 grid columns oriented in a one-dimensional array aligned east-west in each grid column of the GCM. The CSRMs use a horizontal resolution of $4 \mathrm{~km}$, a time step of $20 \mathrm{~s}$, and single-moment microphysics. The semiLagrangian dynamical core was used for large-scale dynamics in SP-CAM, with T42 horizontal resolution, 30 vertical levels, and a time step of $15 \mathrm{~min}$. The embedded CSRMs do not influence the momentum of the global model (i.e., there is no CMT). Compared to the standard CAM, SP-CAM better represents the spectrum of tropical intraseasonal variability (Khairoutdinov et al. 2005; DeMott et al. 2007).

Results are also presented for some integrations of the standard moist CAM, which employed the CAM5 dynamical core (version 5.0). Those integrations used the CAM3 physics package (Collins et al. 2006) to avoid complications introduced by interactive atmospheric aerosols, which are an integral part of the CAM5 physics package. The CAM3 physics includes schemes for deep and shallow/midlevel moist convection (Zhang and McFarlane 1995; Hack 1994). We used the finite volume dynamical core with a resolution of $1.9^{\circ}$ longitude $\times 2.5^{\circ}$ latitude $\times 30$ vertical levels and a time step of $30 \mathrm{~min}$. While the standard CAM thus uses a different dynamical core than SP-CAM, we did repeat some integrations using the SP-CAM dynamical core (i.e., the semiLagrangian core at T42 resolution) with standard CAM convective physics. While there was some sensitivity to the choice of dynamical core, the results were qualitatively similar to those obtained using the CAM5 dynamical core with standard CAM convective physics. All GCMs were integrated for $10 \mathrm{yr}$, with results taken from the last $9 \mathrm{yr}$.

\section{c. Boundary conditions}

All models used an entirely oceanic lower boundary condition with prescribed, zonally symmetric, timeinvariant sea surface temperature (SST). One SST distribution consisted of uniform SST of $300 \mathrm{~K}$. The other is the "Qobs" distribution from the Aquaplanet Experiment (Neale and Hoskins 2001), which is a simple geometric function that approximates the zonal mean of observed SSTs. It is zonally and equatorially symmetric with a maximum of $300 \mathrm{~K}$ on the equator and minima of $273 \mathrm{~K}$ at $60^{\circ} \mathrm{N}$ and $60^{\circ} \mathrm{S}$.

\section{d. Forcings}

Torques in the GCM are identical to those used in SB12 and are illustrated in Fig. 1. These torques are always westward in the upper troposphere and Gaussian in latitude and longitude. They are centered at $90^{\circ} \mathrm{E}$ with standard deviations (i.e., length scales) of $20^{\circ}$ and $10^{\circ}$ in longitude and latitude, respectively. The meridional centers are, in various runs, set at the equator, $10^{\circ} \mathrm{N}$, and $30^{\circ} \mathrm{N}$. Their vertical structure consists of the lognormal distribution given by (4) of SB12, and they have peak amplitudes of $-19 \mathrm{~m} \mathrm{~s}^{-1}$ day $^{-1}$ locally and $-2.5 \mathrm{~m} \mathrm{~s}^{-1} \mathrm{day}^{-1}$ in the zonal mean.

In the full version of the QTCM, the torques consist of a westward forcing for the barotropic mode and a forcing for the baroclinic mode that is westward at upper 

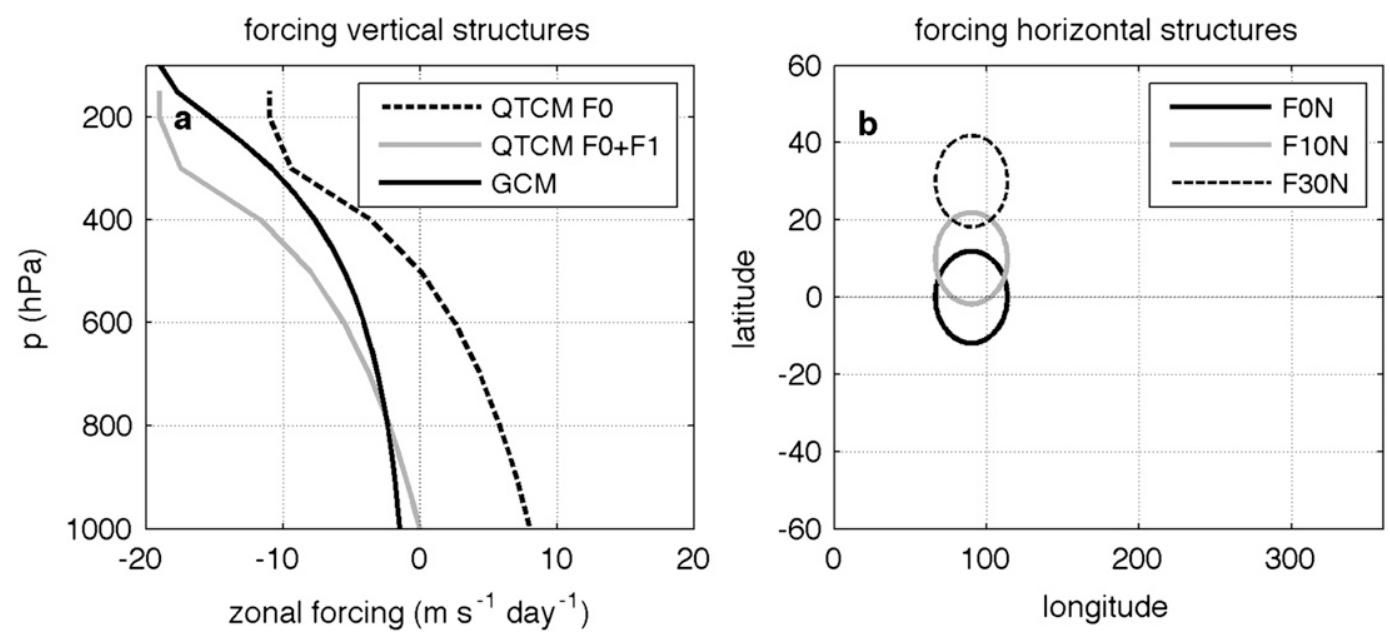

FIG. 1. (a) Vertical structure of the purely baroclinic QTCM forcing (dashed line), the sum of the barotropic and baroclinic QTCM forcings (gray line), and the GCM forcing (solid black line). (b) Horizontal structure of the forcings with contours at half the peak amplitude of the forcings at $0^{\circ}, 10^{\circ}$, and $30^{\circ} \mathrm{N}$.

levels and eastward at lower levels. The amplitudes are chosen so that, when the barotropic and baroclinic forcings are added, the QTCM forcing has the same peak upper-tropospheric amplitude as that used in the GCM. The structure of the barotropic torque is consistent with previous studies that considered single-signed torques (e.g., Haynes et al. 1991; Ring and Plumb 2007; Chen and Zurita-Gotor 2008).

\section{Results}

We present results in order of increasing complexity, starting with the linear version of the QTCM, moving on to nonlinear versions of that model, and finishing with results from GCMs.

\section{a. QTCM response}

In the dry, linear, purely baroclinic version of the QTCM, the upper-level westward torque centered on the equator produces equatorial ascent east of the forcing and subsidence both north and south of the equator west of the forcing (Fig. 2a). As discussed in the introduction and SB12, this pattern can be described by a linear system (e.g., Gill 1980) in which the forcing projects on the ascending phase of an equatorial Kelvin wave east of the forcing and subsiding Rossby wave gyres to the west. Given that this dry, linear QTCM is essentially a numerical version of the analytical model used by SB12, with some modified parameters, it is not surprising that its solutions resemble those analytical results.

We increase complexity gradually, first adding a prognostic moisture equation and convective closure to this model while retaining momentum, moisture, and temperature equations that are otherwise linear and using only one (purely baroclinic) vertical mode. Nonlinearity is included in the QTCM's standard treatment of convection through a Heaviside function that does not permit negative precipitation, and radiation is fully prognostic and depends on moisture. Surface evaporation is fixed at a uniform value of $80 \mathrm{~W} \mathrm{~m}^{-2}$ because allowing it to depend on wind speed would place the model in an unrealistic parameter regime since no barotropic mode exists to destructively interfere with the baroclinic mode near the surface. This moist model produces vertical motion with a horizontal structure nearly identical to that of the dry linear model, but with an amplitude about 3 times larger (Fig. 2b). The horizontal wind response does not increase in amplitude, although it exhibits subtle differences with the dry model. In other words, moisture greatly amplifies the irrotational part of the horizontal flow field while producing only minor changes to the nondivergent part.

These effects can be explained by a reduction of the effective static stability of a moist convecting atmosphere, with adiabatic cooling due to vertical motion partially offset by moist convective diabatic heating (e.g., Emanuel et al. 1994). In the dry, linear QTCM, the steady thermodynamic balance involves the dry static stability $M_{s}$ and a Newtonian cooling operating on time scale $\tau_{r}$ :

$$
M_{s} w_{\mathrm{dry}}=-\frac{T_{1}}{\tau_{r}}
$$

where $T_{1}$ is the temperature anomaly of the single baroclinic mode, $w$ is vertical velocity, and "dry" and "moist" 
subscripts will denote properties of dry and moist models, respectively. ${ }^{1}$ Note that a similar balance would hold even in nonlinear models owing to the weakness of horizontal temperature gradients in the tropics (e.g., Neelin and Held 1987; Sobel and Bretherton 2000). The addition of moisture adds a latent heating term to the right-hand side of (1), which can be eliminated through combination with the moisture equation to produce a steady, linear balance for moist static energy [e.g., section 5c of Neelin and Zeng (2000)]:

$$
\left(M_{s}-M_{q}\right) w_{\text {moist }}=-\frac{T_{1}}{\tau_{r}}
$$

where we neglect variations in surface enthalpy fluxes since those have thus far been fixed at a uniform value. The modified static stability $\left(M_{s}-M_{q}\right)$, which is often called the gross moist stability, is the difference between the dry static stability and the moisture stratification $M_{q}{ }^{2}$ Although the model for which results are shown in Fig. $2 b$ employs a nonlinear moisture closure and a simple radiation scheme instead of Newtonian cooling, (2) seems to explain the amplification of the irrotational response. In particular, $M_{s}$ has a value of about $3.3 \mathrm{~K}$ in temperature units, while $M_{q}$ ranges from 2.1 to $2.4 \mathrm{~K}$ owing to horizontal variations in humidity. The ratio $M_{s} /\left(M_{s}-M_{q}\right)$, which is the factor by which moist convection is expected to amplify vertical velocities, thus ranges from 2.7 to 3.7. Given that the nonlinear moisture closure and interactive radiation can alter the response, this seems close to the ratio of amplitudes of the moist and dry vertical velocities shown in Fig. 2, which ranges from 2.4 to 3.1 .

The zonal-mean mass streamfunction also increases as the effective static stability decreases. This can be seen in the zonal-mean meridional wind, which is linearly related to the QTCM's mass streamfunction and is shown by the gray and dashed blue lines in Fig. 2c for, respectively, the dry and moist integrations just discussed.

\footnotetext{
${ }^{1}$ The QTCM uses vertically integrated equations, so $M_{s}$ is the vertical integral of the product of a standard dry stratification and the vertical structure function of $w$. Both $M_{s}$ and $M_{q}$, a similar vertically integrated quantity for moisture, are formulated in energy units, as is $T_{1}$.

${ }^{2}$ Reduction of the effective static stability by moist convection is a well-known element of theories for tropical atmospheric waves (e.g., Emanuel et al. 1994). But when thinking about the response to a mechanical forcing, one might imagine a "dry" component of vertical motion induced directly by the forcing, with moist convection responding to produce a heating, which in turn causes more ascent and then more heating, ad infinitum. We found that this idea can be formulated in a geometric series that produces the same gross moist stability given in (2).
}
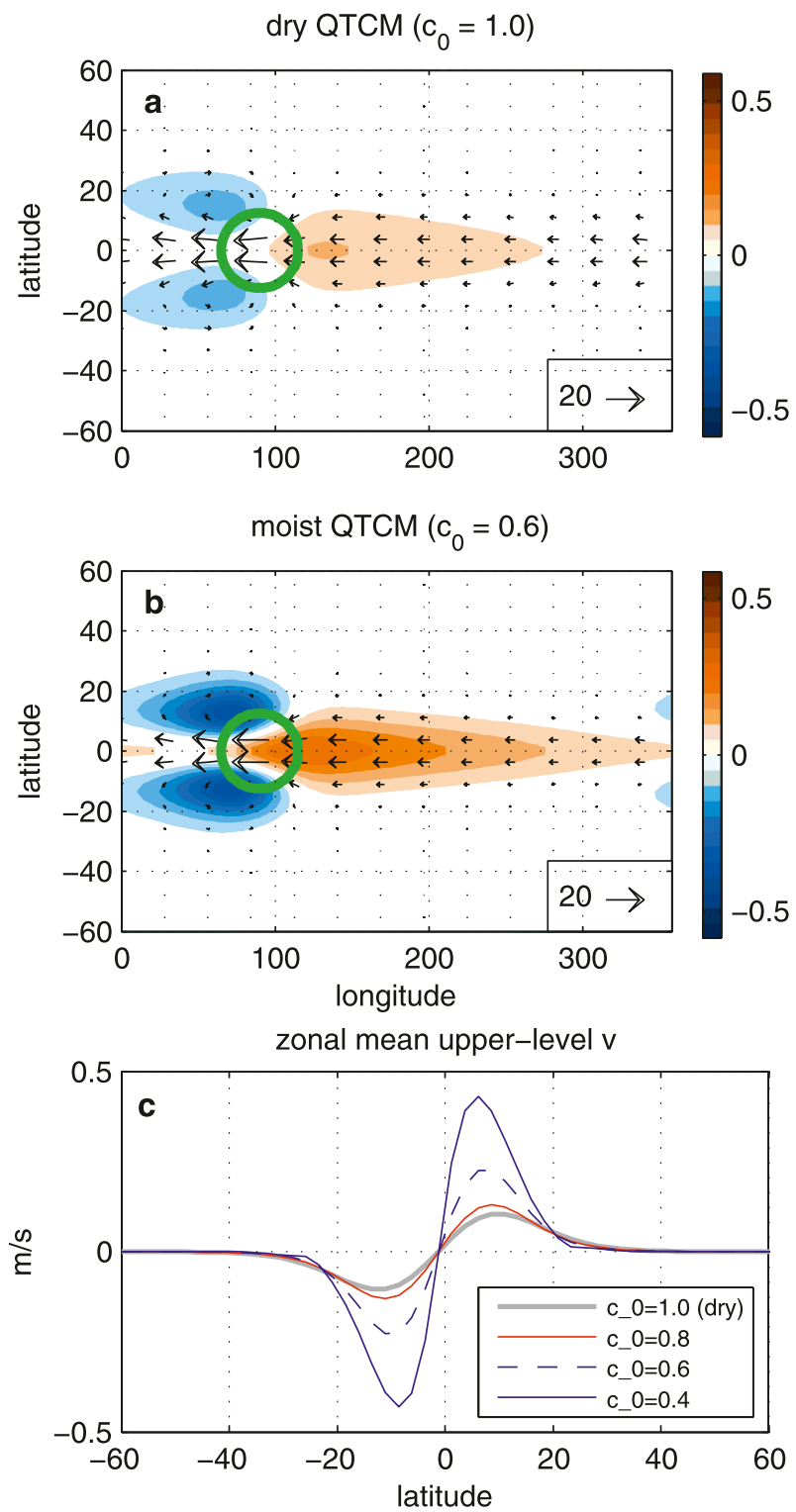

FIG. 2. Response to the equatorial torque of the version of the QTCM with linear dynamics. Results for (a) the dry model and (b) the moist model with uniform surface heat fluxes with colors showing 500-hPa vertical velocity $\left(\mathrm{cm} \mathrm{s}^{-1}\right)$, arrows showing $200-\mathrm{hPa}$ horizontal wind $\left(\mathrm{m} \mathrm{s}^{-1}\right)$, and the green contour marking half the peak amplitude of the forcing. (c) Zonal-mean 200-hPa meridional wind for the dry (gray line) and moist (dashed line) integrations shown in (a) and (b), and for two moist integrations with modified gross moist stabilities (red and solid blue lines). The nondimensional moist gravity wave speed $c_{0}$ is defined in the appendix.

Although the zonal-mean mass flux in the GCMs proves to be constrained by the zonal momentum balance and mostly decoupled from the gross moist stability (see below), the thermodynamic equation exerts a direct influence on the meridional overturning in more highly 

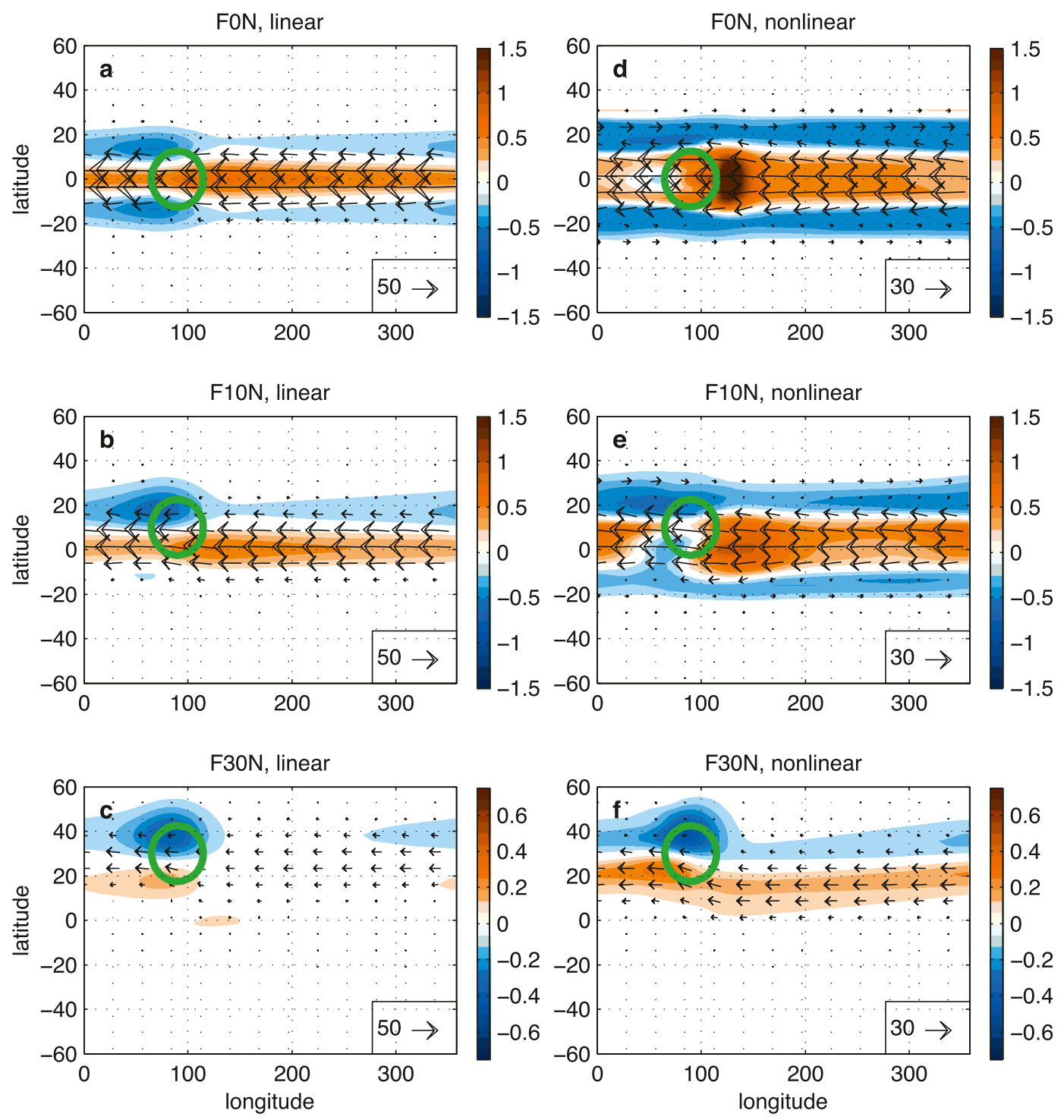

FIG. 3. As in Fig. 2a, but for the response of the moist QTCM to the applied torques. (a)-(c) Linear response and (d)-(f) fully nonlinear response, all with uniform SST and both barotropic and baroclinic modes. Note change in color scale in (c) and (f) and change in reference vector between (left) and (right).

damped models like the QTCM. We consider models with nearly inviscid tropospheres to be better analogs for the real atmosphere, and so explain why the QTCM's zonal-mean overturning is sensitive to the gross moist stability in the appendix.

We next add a barotropic mode to the moist QTCM, but continue to use prescribed, uniform surface heat fluxes and retain linearity by reducing the forcing amplitude by a factor of 100 and multiplying the response by the same factor. As detailed in the previous section, the applied torque projects onto both the barotropic and baroclinic modes. The results essentially replicate those seen in SB12 in a dry GCM (their Fig. 7), except with vertical velocities enhanced by coupling with moist convection. In summary, addition of the barotropic mode greatly increases the amplitude and zonal extent of both the vertical and zonal wind responses (Fig. 3a, cf. Fig. 2b). As the torque is shifted from the equator to $30^{\circ} \mathrm{N}$, subsidence shifts to occur poleward of the subtropical torque in a zonally confined form of the downward-control balance (Figs. 3b, c). In other words, the subtropical forcing is balanced by Coriolis torque on the upper-level meridional flow, with mass continuity maintained by vertical motion at lower levels north and south of the forcing.

The introduction of nonlinearities and interactive surface enthalpy fluxes does not change these qualitative 
characteristics of the response, but does strongly enhance ascent immediately east of tropical torques and increase the meridional scale of the response (Figs. 3d,e). Separate integrations in which wind-induced surface heat exchange (WISHE) and nonlinear advection of momentum, temperature, and moisture were individually suppressed (not shown) reveal that it is temperature and moisture advection that enhance ascent immediately east of the forcing. Nonlinear momentum advection broadens the response meridionally, owing to equatorward transport of eastward momentum by the stationary Rossby wave excited by the torque. WISHE enhances the amplitude and meridional scale of the zonally symmetric component of the $w$ response. For the subtropical torque, WISHE also amplifies ascent and shifts the zonal wind response toward the equator (Fig. 3f), consistent with previous theory for its effects on an off-equatorial ascent zone (Boos and Emanuel 2008). We do not discuss these nonlinearities in further detail here because our equatorial forcings are quite strong and so may overemphasize the importance of nonlinearities, and because the QTCM's large damping produces solutions without transients, which are shown in the next section to be important in GCMs.

All results discussed thus far used a basic state without a meridional temperature gradient or an ITCZ, but amplification of the $w$ response by reduction of the gross moist stability is expected to occur only in precipitating regions. For this reason, we also examine the response to the equatorial torque in the QTCM with a basic-state meridional SST gradient (the Qobs distribution discussed in section 2c). We use a highly simplified moist configuration: a purely baroclinic model without nonlinear advection of momentum, temperature, or moisture, and with radiation represented by a fixed tropospheric radiative cooling. Surface sensible and latent heat fluxes are computed by setting the wind speed used in the model's bulk flux formulas to $8 \mathrm{~m} \mathrm{~s}^{-1}$ everywhere; this allows the SST gradient to affect the circulation while preventing the purely baroclinic dynamics from producing unrealistically large surface enthalpy fluxes. This is similar to the quasi-linear moist model for which results were shown in Fig. 2, but with surface fluxes that allow SST to influence the atmosphere and with simpler radiation that facilitates illustration of a limit on subsidence that we now discuss.

In this simplified QTCM, the response to the equatorial torque in the presence of a meridional SST gradient has many qualitative similarities to the response in the presence of uniform SST (cf. Figs. 4a and 2b). However, the $w$ response seems trapped within the basic-state ITCZ for the nonzero SST gradient. Subsidence west of the torque varies little between $5^{\circ}$ and $20^{\circ}$ of latitude for uniform
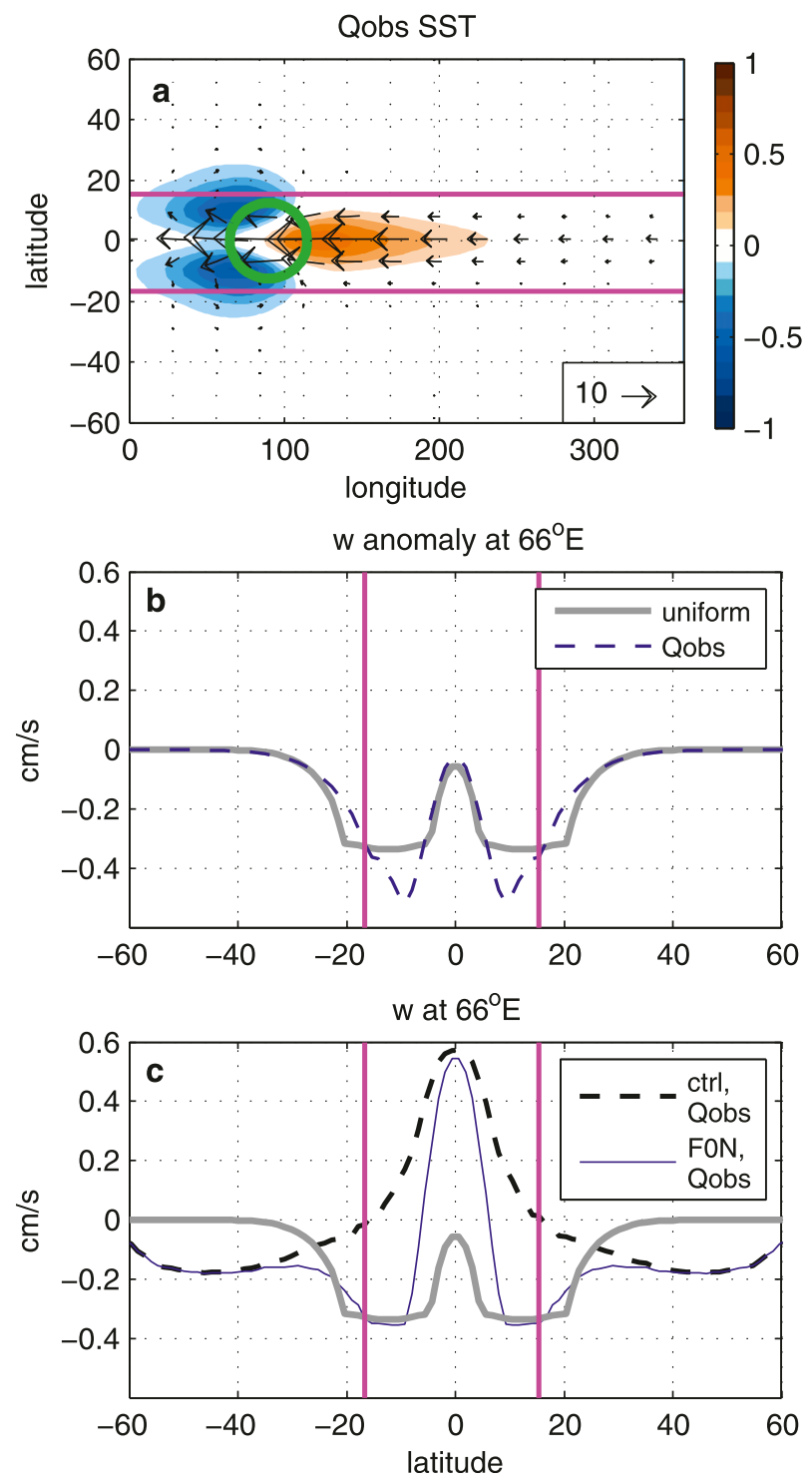

FIG. 4. Quantities are from linear, purely baroclinic moist QTCMs. Magenta lines mark the latitude of the 500-hPa $w=$ 0 line in the basic state with a meridional SST gradient. (a) As in Fig. 2a, but for the response to the equatorial torque in the basic state with a meridional SST gradient. (b) $500-\mathrm{hPa}$ vertical velocity response (i.e., anomaly relative to the basic state) at $66^{\circ} \mathrm{E}$ with a meridional SST gradient (dashed line) and with uniform SST (gray line). (c) Total $500-\mathrm{hPa}$ vertical velocity at $66^{\circ} \mathrm{E}$ for the model with a meridional SST gradient and no torque (dashed line), for that same model with the equatorial torque (thin blue line), and for the model with uniform SST forced by the equatorial torque [gray line, as in (b)].

SST, while in the presence of a nonzero SST gradient the subsidence response peaks sharply within the basic-state ITCZ (Fig. 4b). No sharp peaks in the subsidence field are seen when one examines the total $w$ (blue line in Fig. 4c) rather than the anomaly relative to the control, and the 
total $w$ seems to saturate near the same value of $-0.3 \mathrm{~cm} \mathrm{~s}^{-1}$ seen in the integration with uniform SST. This apparent limit corresponds to the value of subsidence needed to produce the adiabatic warming that balances clear-sky radiative cooling. For $M_{s}=3.3 \mathrm{~K}$ and the imposed atmospheric radiative flux divergence of $80 \mathrm{~W} \mathrm{~m}^{-2}$, this limit is $-0.33 \mathrm{~cm} \mathrm{~s}^{-1}$ at $500 \mathrm{hPa}$ in the QTCM-quite close to the value at which subsidence seems to saturate in the model. The apparent trapping of the response within the basic-state ITCZ thus results from this lower bound on $w$ combined with calculation of the response by subtraction of the basic-state $w$. The total forced subsidence west of the torque exhibits some dependence on SST (e.g., compare blue and gray lines in Fig. 4c) because surface sensible heat fluxes influence the thermodynamic balance that constrains $w$. Although meridional trapping of the $w$ response by the basic-state ITCZ is a somewhat subtle effect in the QTCM, it is more pronounced in the GCMs (see below) and is discussed here because the QTCM provides a simple framework in which to explore the dynamics.

One next step could be examination of the response to torques in a basic state with a meridional SST gradient using the full, nonlinear, moist QTCM. However, SB12 showed that the dry response in baroclinic basic states involves large changes in meridional momentum fluxes associated with extratropical baroclinic eddies. The QTCM was not designed to faithfully represent such eddies, and its relatively strong damping may limit its ability to represent responses that involve free-tropospheric Hadley circulation dynamics. So we turn to GCMs to more thoroughly examine the moist response to torques.

\section{b. GCM response, barotropic basic state}

The torques shown in Fig. 1 are applied in the dry and moist GCMs with barotropic basic states, and the response of the upper-tropospheric horizontal wind and midtropospheric vertical wind are shown in Fig. 5. ${ }^{3}$ Both dry and moist solutions exhibit equatorial ascent and off-equatorial subsidence in response to tropical torques. There is the familiar shift to a zonally confined downward-control balance for the $30^{\circ} \mathrm{N}$ torque, with peak subsidence poleward of the torque.

The peak amplitude of the $w$ response is several times larger in the moist GCM (SP-CAM) than in the dry GCM. This is consistent with a reduced effective static stability in the moist model, although moisture introduces

\footnotetext{
${ }^{3}$ The dry response to the equatorial torque is identical to that shown in SB12, but SB12 did not show the dry response to offequatorial torques in a barotropic basic state with a nearly inviscid free troposphere.
}

modifications that are more complicated than a simple increase in amplitude of the $w$ response. Nevertheless, peak vertical velocities scale as one might expect based on a simple reduction of gross moist stability. Peak ascent for tropical torques is about 3 times larger in SP-CAM than in the dry GCM, while the static stability $\partial_{p} \theta$ in the dry GCM is about one-third as large as in SP-CAM (we use a conventional stability for the GCMs instead of the vertically integrated quantity $M_{s}$ used in the QTCM). If one assumed the $w$ response to a torque simply scaled like $1 / \partial_{p} \theta$ [or like $1 / M_{s}$ as in (1)], then one would obtain peak vertical velocities in the moist model that are about one-third of those in the dry model. However, the moist model produces values about 3 times those of the dry model, so the diabatic effects of moisture seem to enhance peak ascent by a factor near 9 . While this is larger than the factor of 3 difference in the gross moist and dry stabilities in the QTCM, it is roughly consistent with estimates of the ratio of gross moist to dry stabilities in the observed tropical troposphere (e.g., Yu et al. 1998). Given that the gross moist stability of a GCM can be modified by tuning its moist convection scheme (Frierson 2007), one would not expect a precise agreement between this moist GCM and either observations or other models.

Additional insight on the influence of the gross moist stability can be obtained from the divergence budget (e.g., Stevens 1979). SB12 showed that the response to the torque in dry models can be described by the two parts of the divergence budget,

$$
\frac{\partial^{2} \Phi}{\partial x^{2}} \simeq \frac{\partial \mathcal{F}}{\partial x}
$$

and

$$
f \frac{\partial u}{\partial y}+\beta u \simeq-\frac{\partial^{2} \Phi}{\partial y^{2}},
$$

where $\mathcal{F}$ is the applied torque, $\Phi$ is the geopotential, other symbols have standard meteorological meanings, and Cartesian coordinates are used for purposes of discussion. These are obtained from the zonal and meridional momentum equations, respectively, and express approximate balances seen in the dry GCMs of SB12. The westward torque forces upper-level divergence to its east and convergence to its west, and the forcing is balanced in the steady state by zonal curvature of $\Phi$, as in (3). This zonal deformation of $\Phi$ can be seen in the moist and dry GCMs, with a minimum of $\Phi$ east of the torque and a maximum to the west (Fig. 6a). In the tropical free troposphere, forcings that induce gradients in $\Phi$ produce vertical motions that act to rapidly 

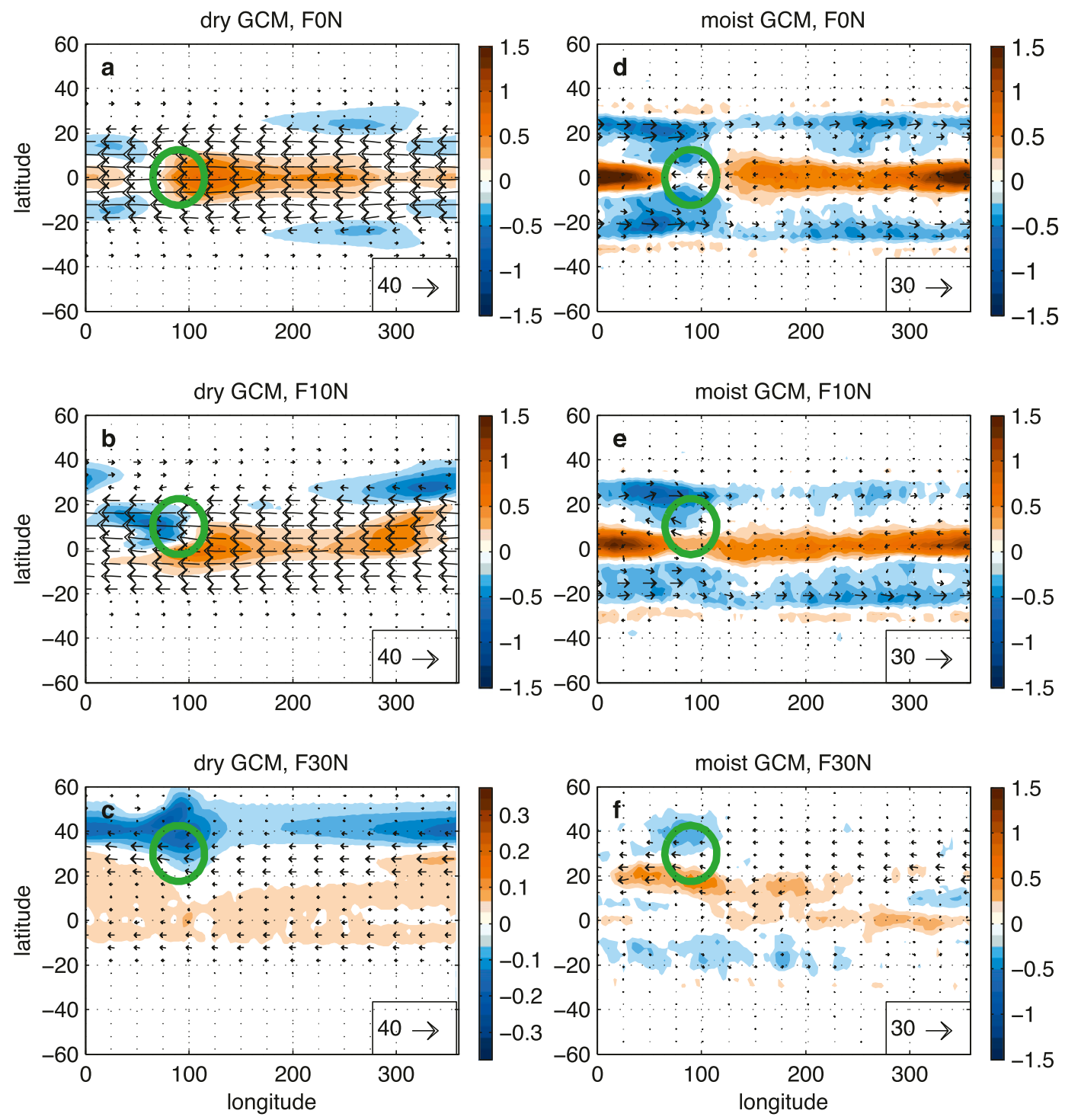

FIG. 5. (a)-(c) Solutions from dry GCM integrations and (d)-(f) from moist SP-CAM integrations with uniform SST. Colors show 500-hPa vertical velocity $\left(\mathrm{cm} \mathrm{s}^{-1}\right)$, arrows show $200-\mathrm{hPa}$ horizontal wind $\left(\mathrm{m} \mathrm{s}^{-1}\right)$, and the green contour marks half the peak amplitude of the forcing. Note change in color scale in (c) and change in reference vector between (left) and (right).

homogenize $\Phi$ owing to the large Rossby deformation radius, and these vertical motions increase in amplitude as the gross moist stability decreases. This explains the strong amplification of the equatorial Kelvin wave response in the moist GCMs relative to the dry GCM. However, the balance (3) is only approximate; the divergent and rotational parts of the response cannot be entirely separated, as evidenced by the dominant terms in the vorticity budget (shown in Fig. 6b),

$$
f \delta+\mathbf{u} \cdot \nabla(\zeta+f) \simeq-\frac{\partial \mathcal{F}}{\partial y},
$$

where $\delta$ is the divergence of the horizontal wind $\mathbf{u}$ and $\zeta$ is the relative vorticity. This balance is also consistent with the dynamics discussed by SB12. The forcing $\partial_{y} \mathcal{F}$ is locally balanced by vorticity advection accomplished mostly by rotational flow, but in tropical regions away from the torque vorticity advection is balanced by the stretching term.

Although reductions in the gross moist stability amplify part of the divergent response [e.g., that associated with vertical motions that relax the equatorial $\Phi$ gradients given by (3)], meridional divergent circulations are forced only indirectly by $\mathcal{F}$ and are governed by 

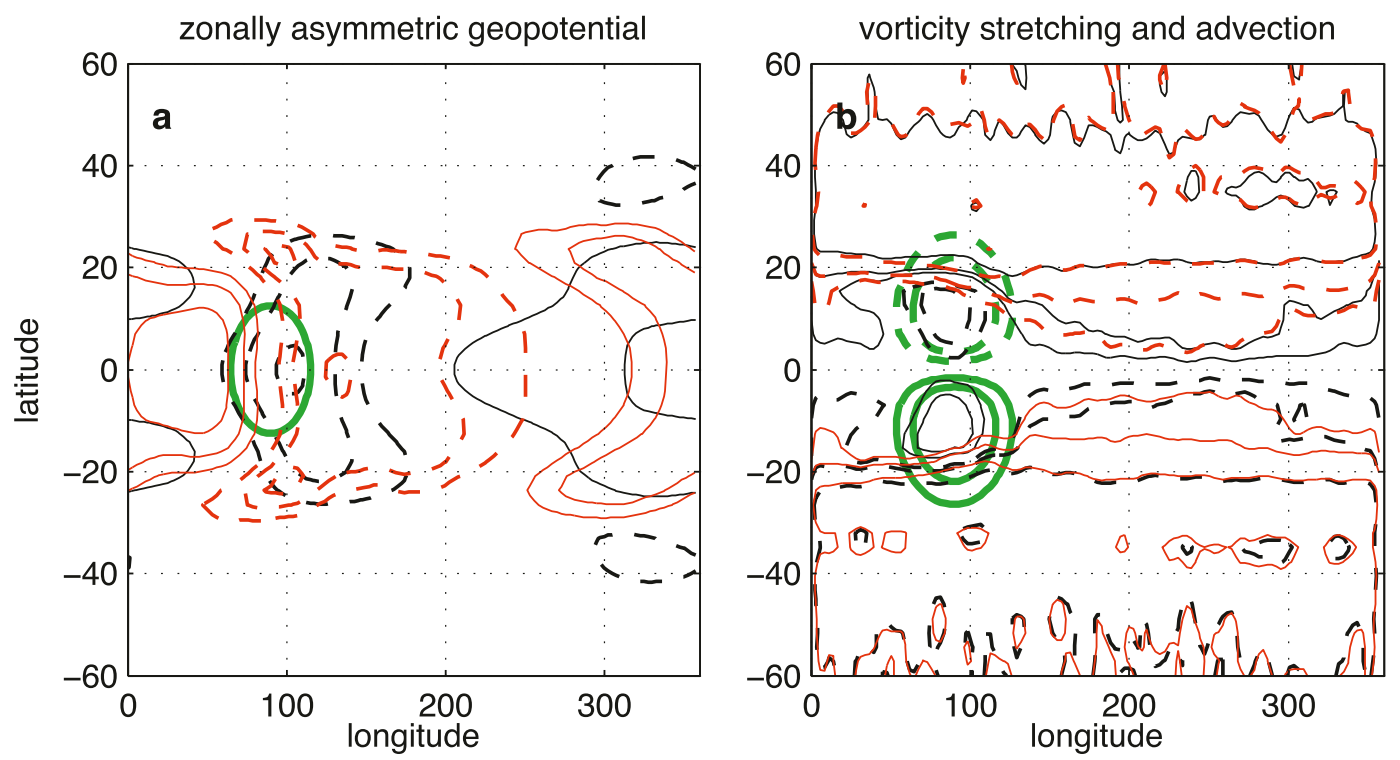

FIG. 6. (a) Zonally asymmetric component of the 200-hPa geopotential height in the dry GCM (black) and in the moist GCM (SP-CAM; red). Contour interval is $10 \mathrm{~m}$ with the zero contour not shown, negative contours dashed, and the torque outlined in green at half its peak value. (b) Dominant terms in the 200-hPa vorticity budget for the moist GCM (SP-CAM). Black contours show horizontal advection of absolute vorticity, red contours show vorticity stretching (the product of divergence and absolute vorticity), and green contours show the meridional gradient of the torque, with each term having the sign given in (5). Contour magnitudes are $[2,4,10] \times 10^{-11} \mathrm{~s}^{-2}$ with negative contours dashed. All quantities are time means of daily total fields for integrations with the equatorial torque.

different constraints. This can be seen in (4), which is the part of the divergence budget associated with tendencies of $\partial_{y} v$ and which shows that these tendencies depend on geostrophically balanced zonal winds, rather than the torque itself. Zonal winds differ greatly between the moist and dry GCMs, with the former having much weaker equatorial westward flow (Fig. 7). Thus, it is not surprising that peak values of the zonal-mean mass streamfunction $\Psi$ do not scale with the moist stability in the same way as the peak $w$ response (Fig. 7). Differences in the $\Psi$ maxima are small compared to differences in the peak $w$ response and compared to the difference between the dry and moist $\Psi$ maxima in the QTCM (Fig. 2c; see appendix for discussion on $\Psi$ in the QTCM).

A useful constraint on $\Psi$ is found in the approximate zonal-mean $u$ balance for the upper troposphere,

$$
-\beta y \bar{v}+\bar{v} \frac{\partial \bar{u}}{\partial y}+\bar{w} \frac{\partial \bar{u}}{\partial z} \simeq \overline{\mathcal{F}}+\bar{S},
$$

where symbols have usual meteorological meanings and an overbar denotes a zonal mean. The torque $\overline{\mathcal{F}}$ modifies $\bar{v}$ via a balance between the Coriolis force and $\overline{\mathcal{F}}$ [as discussed by Richter and Rasch (2008)], with additional contributions from the eddy momentum flux convergence $\bar{S}$ and nonlinear momentum advection by the time-mean, zonal-mean meridional circulation (MMC). In particular, near-equatorial torques induce eddy momentum flux convergence (EMFC) in the equatorial upper troposphere that is of similar amplitude but opposite sign to the applied torque but also of more limited spatial extent (Fig. 8; EMFC is calculated from daily deviations about the zonal mean and includes vertical and horizontal transports). For example, the applied torque peaks at $-2.5 \mathrm{~m} \mathrm{~s}^{-1}$ day $^{-1}$ in the zonal mean, while the zonal-mean EMFC response to this torque peaks at $2.6 \mathrm{~m} \mathrm{~s}^{-1}$ day $^{-1}$ in the dry GCM and $3.3 \mathrm{~m} \mathrm{~s}^{-1}$ day $^{-1}$ in SP-CAM. But the EMFC is limited to a much smaller region of the tropical upper troposphere than the applied torque (cf. orange shading with the dashed green contour in Fig. 8). The EMFC is thus not constrained to balance the applied torque, and instead has a structure that suggests it is a particular response of the equatorial upper troposphere.

Much of the difference in $\Psi$ between the dry and moist simulations can be explained in terms of the EMFC. The part of $\Psi$ that balances the EMFC can be diagnosed from the steady, inviscid $u$ balance (e.g., Haynes et al. 1991; Seager et al. 2003):

$$
\Psi_{e}(\phi, p)=\frac{2 \pi a \cos \phi}{g f} \int_{p}^{0} \bar{S} d p^{\prime} .
$$

For equatorial torques, $\Psi_{e}$ cancels part of the linear response to the torque within about $10^{\circ}$ of the equator, 

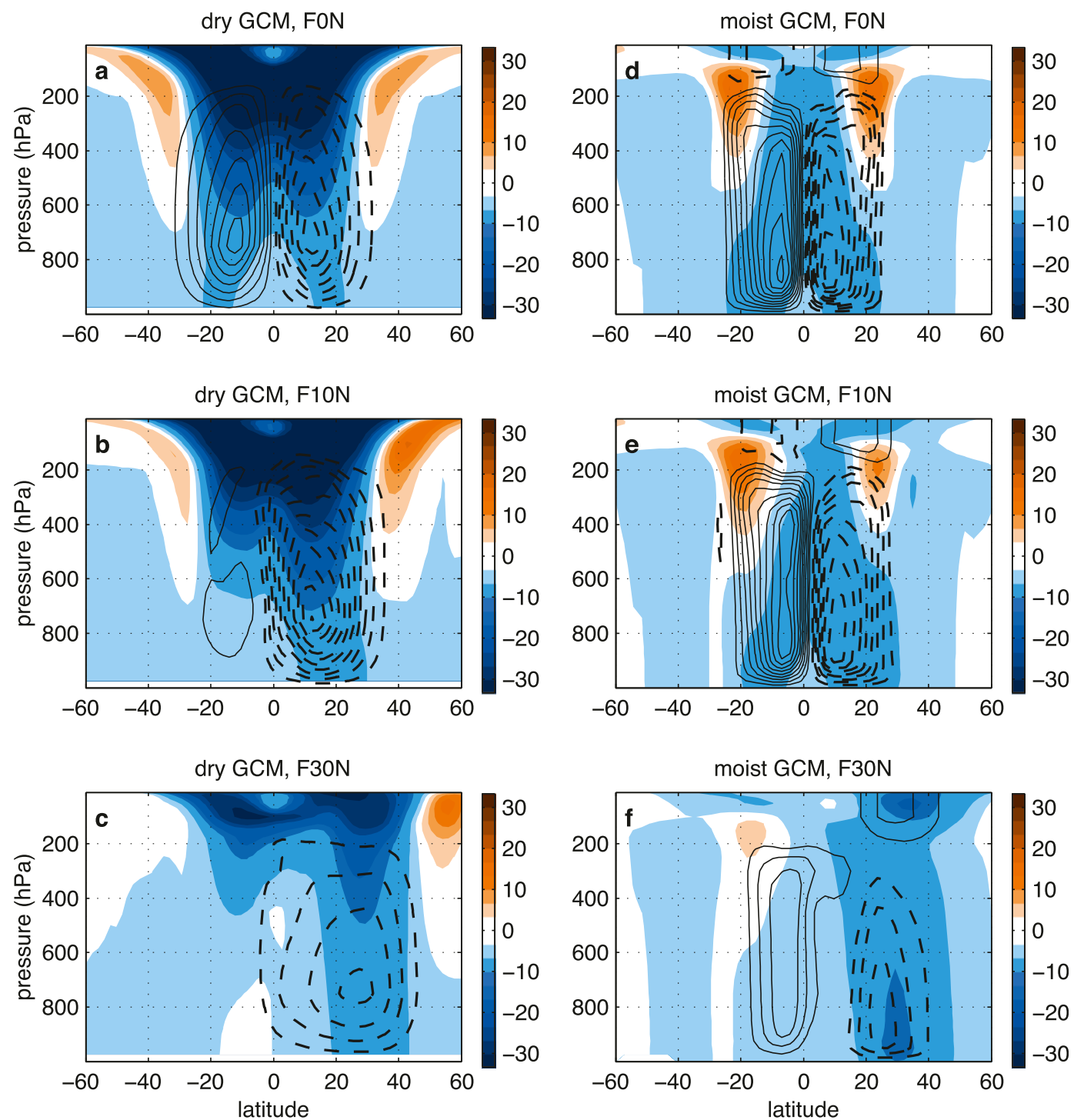

FIG. 7. As in Fig. 5, but showing the zonal-mean mass streamfunction (contours, negative dashed, and interval $10^{10} \mathrm{~kg} \mathrm{~s}^{-1}$ ) and zonal-mean zonal wind (colors, $\mathrm{m} \mathrm{s}^{-1}$ ).

but enhances the linear response near and poleward of $20^{\circ}$ latitude (Fig. 8), which broadens $\Psi$ meridionally. For the $10^{\circ} \mathrm{N}$ torque, the Southern Hemisphere EMFC persists in the moist model and seems to explain a large fraction of the strong Southern Hemisphere overturning that is seen in the moist model but not in the dry (middle panels of Figs. 7 and 8). A Southern Hemisphere response persists in the moist model even for the $30^{\circ} \mathrm{N}$ torque. However, the Southern Hemisphere $\Psi_{e}$ shown in Fig. $8 \mathrm{f}$ accounts for less than half the magnitude of the total $\Psi$ response seen in Fig. 7f.

The apparent strong coupling of the EMFC to $\Psi$ motivates examination of the nature of the EMFC. In both the dry and moist GCMs with barotropic basic states, the EMFC comprises almost entirely transient, horizontal EMFC (not shown). This is in sharp contrast to the QTCM, for which solutions with barotropic basic states contained negligible transients and tropical EMFC was accomplished by stationary eddies.

Transient EMFC in the moist models is associated with a red spectrum of convectively coupled equatorial motions dominated by an eastward-propagating, wavenumber-1 disturbance. This can be seen in the wavenumber-frequency power spectrum of the equatorially symmetric component of 500-hPa $w$ (Fig. 9a), which is qualitatively similar to the spectrum of the equatorially antisymmetric component of upper-tropospheric $v$ (not shown). These power spectra were computed for winds 

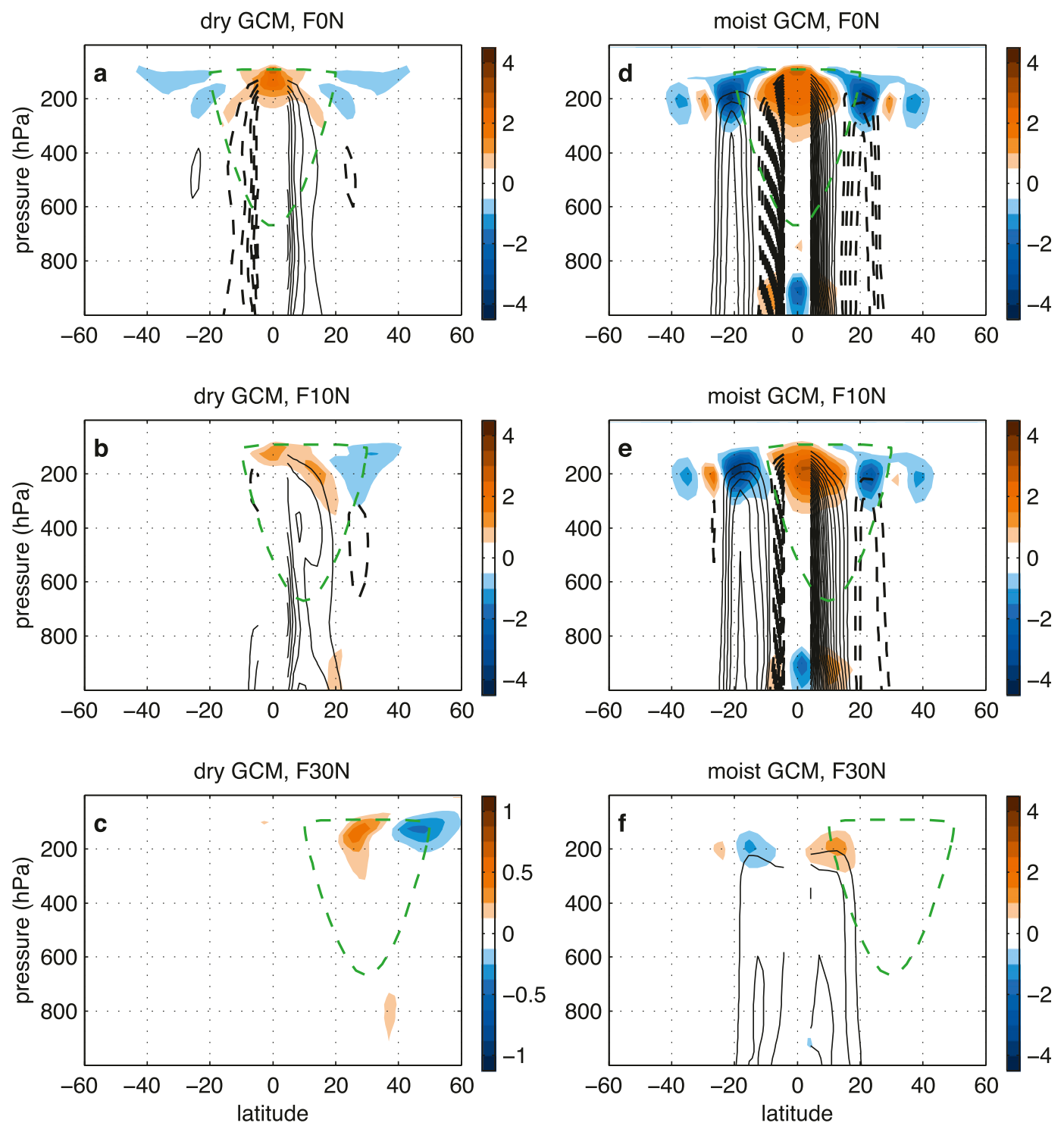

FIG. 8. As in Fig. 5, but showing the EMFC (colors, $\mathrm{m} \mathrm{s}^{-1}$ day $^{-1}$ ) and the part of the zonal-mean mass streamfunction that balances the EMFC (contours, negative dashed, and interval $10^{10} \mathrm{~kg} \mathrm{~s}^{-1}$ ). Dashed green line marks the location where the zonal-mean forcing is $-0.5 \mathrm{~m} \mathrm{~s}^{-1} \mathrm{day}^{-1}$, which is the same amplitude as the weakest color shading (and differs from the solid green contours shown in Figs. 2-5).

within $10^{\circ}$ of the equator using the methodology of Wheeler and Kiladis (1999) as implemented in the NCAR Command Language (NCL) version 5.1.0. The equatorial torque increases spectral power in the moist model by about half an order of magnitude over a broad range of wavenumbers and frequencies corresponding to westward-propagating disturbances (Fig. 9c). The lowest-frequency eastward-propagating disturbances are also strongly enhanced, and the fact that these disturbances dominate the spectrum of the basic state should be noted when interpreting the right column of Fig. 9 (i.e., the absolute increase in power of the low-frequency eastward-propagating disturbances is larger than that of the high-frequency westwardpropagating disturbances, even though the relative change in the former is smaller). The increase in variance of westward-propagating disturbances occurs at phase speeds considerably faster than that of $\bar{u}$, indicating the spectrum is not simply Doppler shifted by the induced mean flow (cf. the phase speed line in Fig. 9c with $\bar{u}$ in Fig. 7d).

In contrast to this general modification of the equatorial wave field in the moist GCM, spectra for the dry GCM show power concentrated around a wavenumber- 4 

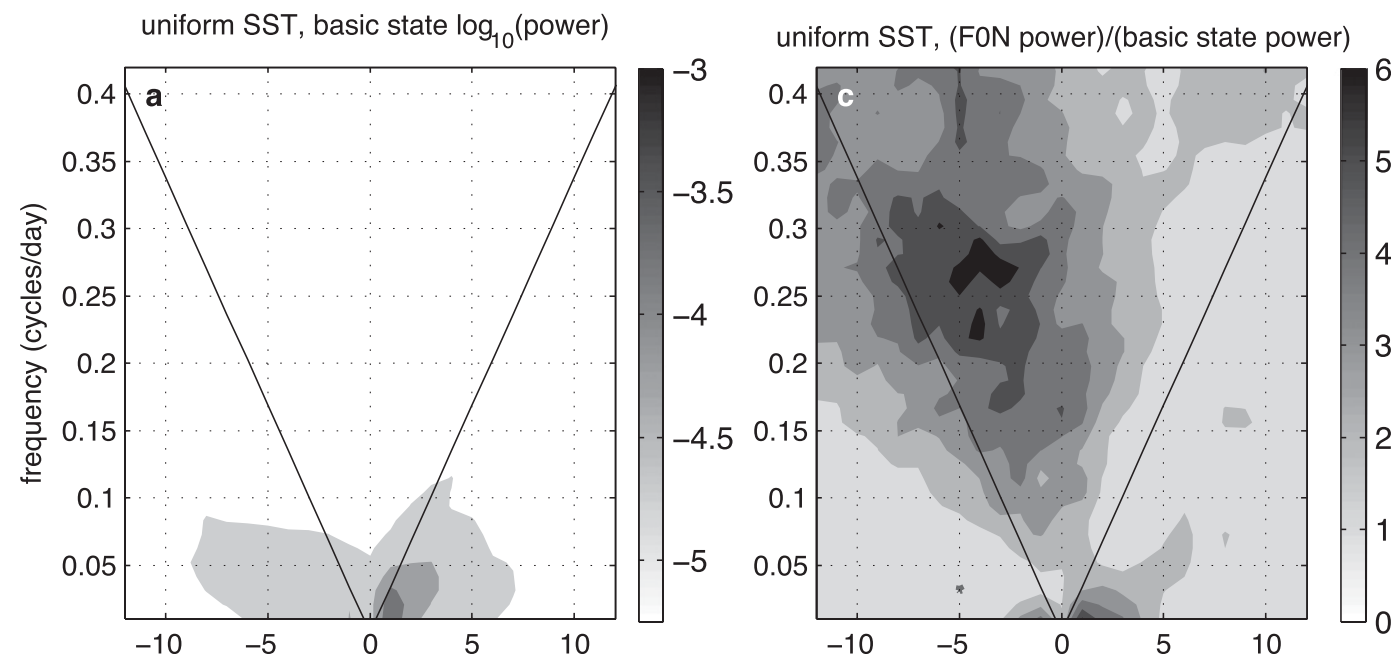

qobs SST, basic state $\log _{10}$ (power)

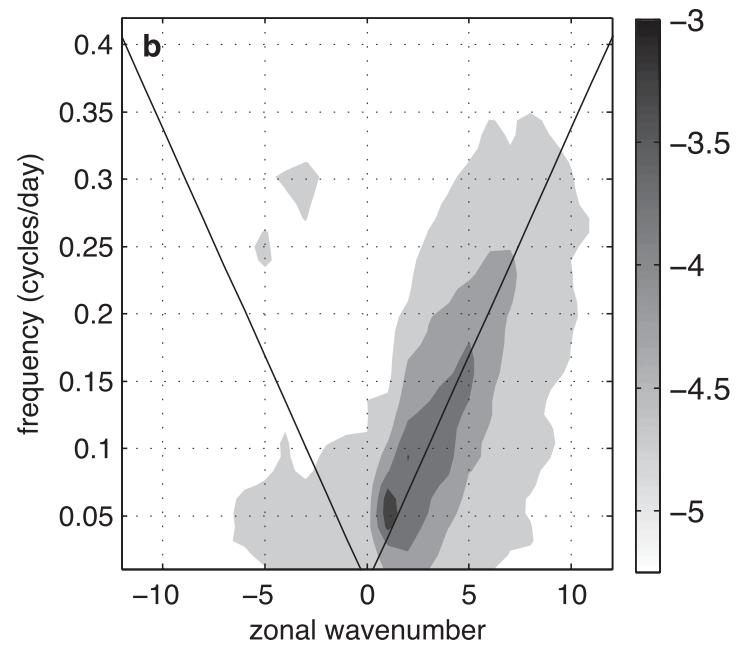

qobs SST, (FON power)/(basic state power)

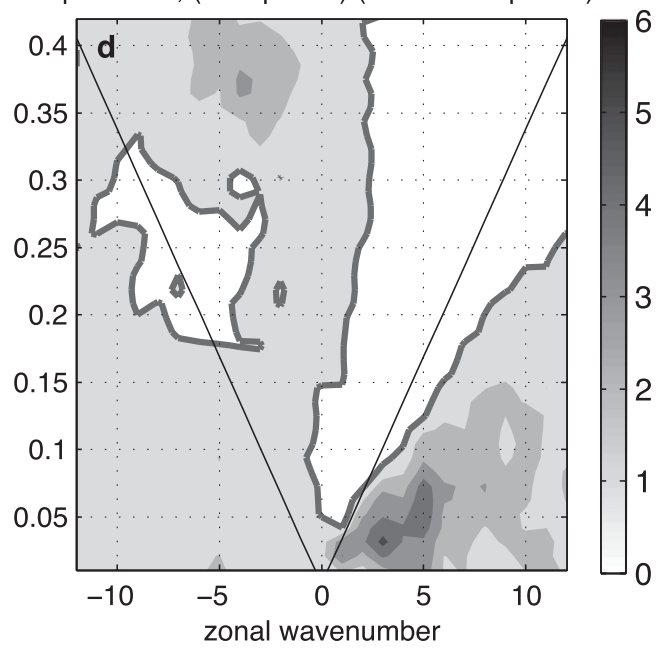

FIG. 9. Power spectra, as a function of zonal wavenumber and frequency, of the equatorially symmetric component of $500-\mathrm{hPa}$ vertical velocity averaged over $10^{\circ} \mathrm{N}-10^{\circ} \mathrm{S}$ for SP-CAM. Results for (a),(c) the basic state with uniform SST and (b),(d) the basic state with a meridional SST gradient. (left) The base-10 logarithm of power in the basic state and (right) the ratio of power in integrations forced by the equatorial torque to power in the basic state. Thin solid lines show shallow-water Kelvin wave phase speeds of $16 \mathrm{~m} \mathrm{~s}^{-1}$ (25-m equivalent depth), and thick gray line in (d) is the zero contour.

disturbance propagating westward at $35 \mathrm{~m} \mathrm{~s}^{-1}$ (not shown), which is near the upper-level $\bar{u}$ in that model (Fig. 7a). This, and the fact that these transient eddies have a dynamical structure indicative of equatorial Rossby waves confined to the upper troposphere, is consistent with the suggestion of SB12 that barotropic instability contributes to upper-level EMFC in the dry model, with the resulting Rossby waves advected westward by the mean flow. Given these differences in the properties of transients in the dry and moist models, it is not surprising that the EMFC response has a different amplitude and spatial structure in the two models. Theories for the destabilization of free equatorial convectively coupled waves are an active topic of research [see Kiladis et al. (2009) for a review], and similar mechanisms may apply to forced waves. Regardless of the detailed wave mechanisms, it seems clear that tropical torques force equatorial ascent and amplify equatorial convectively coupled waves in the barotropic basic state of SP-CAM; like steady equatorial heatings, those waves converge momentum onto the equator (e.g., Suarez and Duffy 1992; Norton 2006) and are associated with secondary meridional circulations.

Nonlinear momentum advection by the MMC is also important. The ratio of the local vertical component of relative vorticity to the Coriolis parameter peaks at 

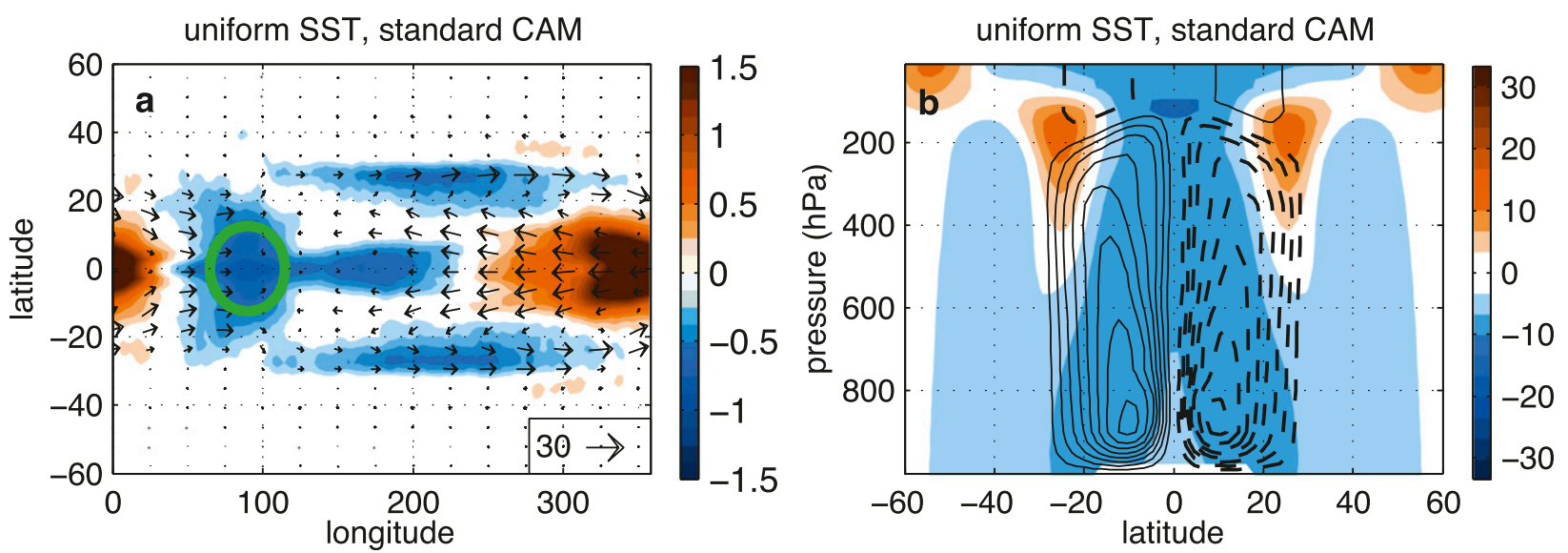

FIG. 10. Response to the equatorial torque in the standard moist CAM with uniform SST. (a) As in Fig. 5 and (b) as in Fig. 7.

0.5 near $10^{\circ}$ latitude in the upper troposphere of most runs with torques (not shown), indicating that both EMFC and nonlinear advection by the MMC are important in the $\bar{u}$ balance (e.g., Walker and Schneider 2006). For the equatorial torque in particular, symmetry dictates that advection by the MMC must be negligible precisely on the equator near the upper boundary of the induced circulation so that $\overline{\mathcal{F}}$ must be balanced entirely by $\bar{S}$ there, consistent with the location of maxima of $\bar{S}$ in both the dry and moist GCMs (Fig. 8). In contrast, $\bar{S}$ is small in the midtroposphere so that $\overline{\mathcal{F}}$ must be balanced there by nonlinear momentum advection by the MMC. The fact that the equatorial maximum of $\bar{S}$ is substantially weaker in the dry GCM than in the moist GCM is consistent with the larger equatorial vertical shear of $\bar{u}$ in the dry GCM, which allows vertical momentum advection to balance $\overline{\mathcal{F}}$.

The response to equatorial torques is sensitive to model details, in particular to the representation of moist convection, which is not surprising given the effects of the torque on convectively coupled equatorial waves and their associated EMFC. Compared to SP-CAM, the standard moist CAM produces ascent in response to the equatorial torque that is meridionally broader and more zonally confined (cf. Figs. 10a and 5d). Subsidence, rather than ascent, occurs immediately east of the forcing in the standard CAM, and upper-level eastward flow is seen in the region of the westward forcing. The EMFC response to the torque has a peak amplitude almost twice as large as that in SP-CAM, but is otherwise highly similar in its vertical and meridional structure (not shown). Although the peak zonal-mean mass streamfunction response is about $20 \%$ weaker in the standard CAM than in SP-CAM, it has a similar meridional width and structure (cf. Figs. 10b and 7d). We do not endeavor to understand the reasons for all these differences, as they likely depend on details of each model's representation of convectively coupled equatorial waves.

The response to the $30^{\circ} \mathrm{N}$ torque seems less sensitive to model details than the response to tropical torques. Both SP-CAM and the QTCM show ascent just equatorward and subsidence just poleward of the $30^{\circ} \mathrm{N}$ torque, consistent with the zonally localized downward-control-type balance discussed in SB12 (bottom panels of Figs. 3 and 5). The response to the $30^{\circ} \mathrm{N}$ torque is also highly similar in the standard CAM (not shown). The amplitude of the $w$ response is locally stronger in the moist than in the dry GCM but $\Psi$ has nearly the same peak value in both models (Figs. 7c,f), consistent with the fact that the extratropical EMFC response is negligibly small in both models. The $30^{\circ} \mathrm{N}$ torque produces subsidence in the Southern Hemisphere only in the moist GCMs, as discussed above in connection with the EMFC response.

\section{c. GCM response, baroclinic basic state}

We next present results for moist GCMs using a basic state with a meridional SST gradient. This basic state has equatorially antisymmetric Hadley and Ferrel cells and symmetric westerly jets (Fig. 11). Compared to the standard CAM basic state, SP-CAM has slightly stronger tropical streamfunction extrema, a narrower ITCZ, and stronger westerly jets that are positioned closer to the equator. The westerly jet in the standard CAM is positioned in the upper branch of the Ferrel cell, within the region of positive extratropical EMFC (Fig. 11). In contrast to that eddy-driven jet, the SP-CAM westerly jet has a weaker surface amplitude and is more nearly in quadrature with the basic-state EMFC, so seems more "subtropical" and less eddy driven in nature. The wider 

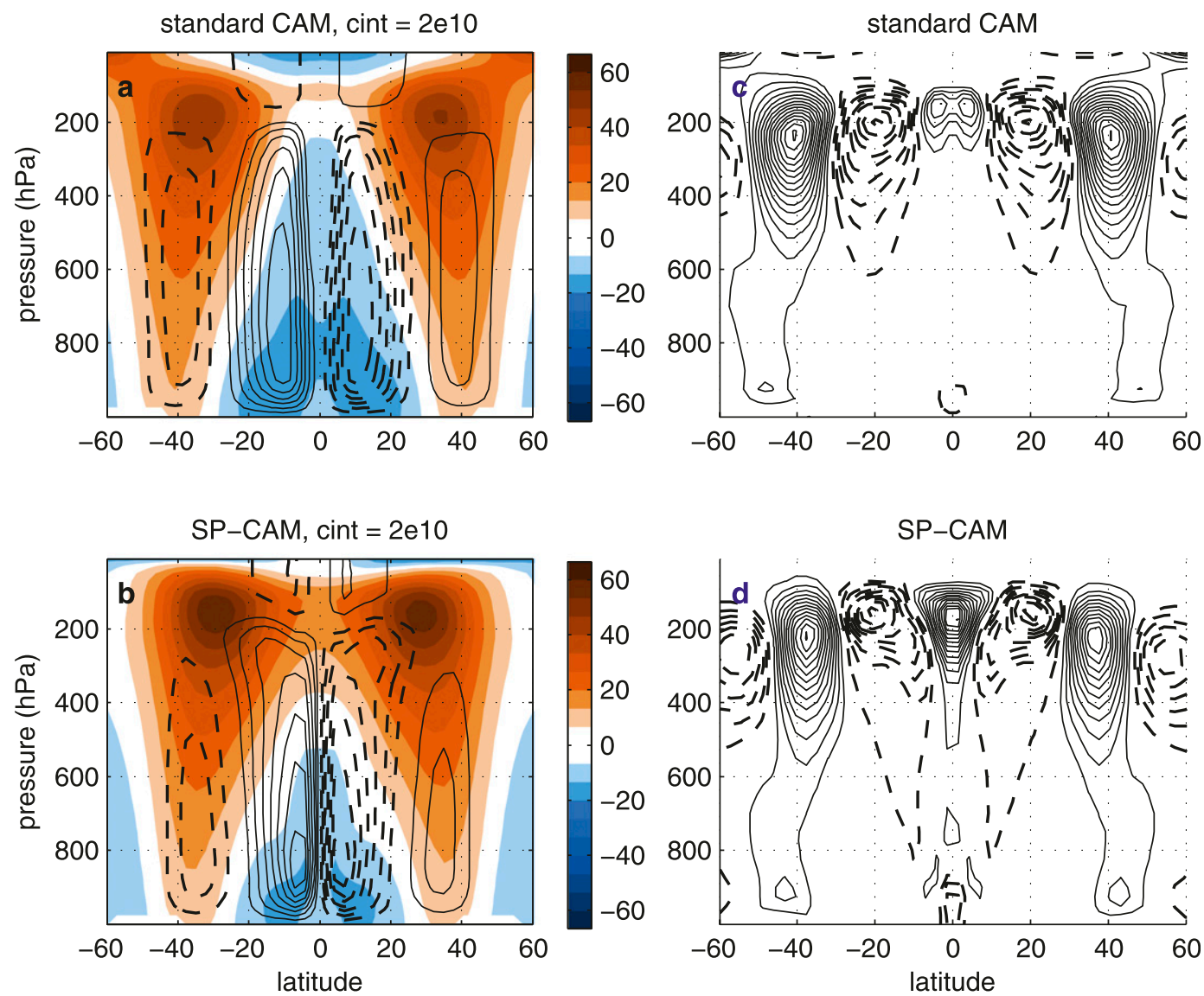

FIG. 11. Properties of the basic state with a meridional SST gradient for the (a),(c) standard moist CAM and (b),(d) SP-CAM. (left) Zonal-mean mass streamfunction (contours, interval $2 \times 10^{10} \mathrm{~kg} \mathrm{~s}^{-1}$ with negative dashed) and zonal-mean zonal wind (colors, $\mathrm{m} \mathrm{s}^{-1}$ ). (right) Zonal-mean EMFC (contour interval $0.5 \mathrm{~m} \mathrm{~s}^{-1}$ day $^{-1}$ with negative dashed).

ITCZ in the standard CAM is actually a double ITCZ, as seen in the distribution of zonal-mean precipitation (dashed line in Fig. 12b). Unlike the baroclinic basic state of the dry GCM presented in SB12, both of these moist GCMs exhibit equatorial super rotation with positive EMFC in the equatorial upper troposphere.

The response to tropical and subtropical torques in this basic state has high amplitude in both the tropics and extratropics. For all three torques, most of the tropical response is confined within the basic-state ITCZ, as delineated by the $500-\mathrm{hPa} w=0$ contours in the SPCAM basic state (Fig. 13). A similar phenomenon was discussed in the context of the QTCM, where we argued that the $w$ response is amplified within the basic-state ITCZ by the ratio $M_{s} /\left(M_{s}-M_{q}\right)$, while outside the basic-state ITCZ the subsidence response saturates when adiabatic warming by total subsidence balances clearsky radiative cooling (e.g., Fig. 4). This effect is more prominent in the moist GCMs which, as noted in the previous section, seem to have a larger difference between dry and gross moist stabilities than the QTCM. A meridional section of 500-hPa $w$ slightly west of the torque $\left(40^{\circ}-\right.$ $110^{\circ} \mathrm{E}$ ) looks similar to that seen in the QTCM, with total subsidence west of the torque roughly constant between $5^{\circ}-30^{\circ}$ latitude and the sharp, near-equatorial extrema appearing only after subtraction of the basic-state $w$ (Fig. 14). Subsidence near $30^{\circ} \mathrm{N}$ and $30^{\circ} \mathrm{S}$ does increase in amplitude beyond that in the basic state for the tropical torques, but this occurs in a region of strong meridional temperature gradient and equatorward flow, indicating that horizontal temperature advection balances some of the enhanced adiabatic warming in this case.

Eddy momentum fluxes modify the zonal-mean streamfunction response in ways quite different from those seen in models with barotropic basic states. The EMFC response in SP-CAM is dominated by extratropical features associated with a poleward shift in the basic-state EMFC (Fig. 15; compare with the basicstate EMFC in Fig. 11 and the EMFC response for the barotropic basic state in Fig. 8). This is similar to the 

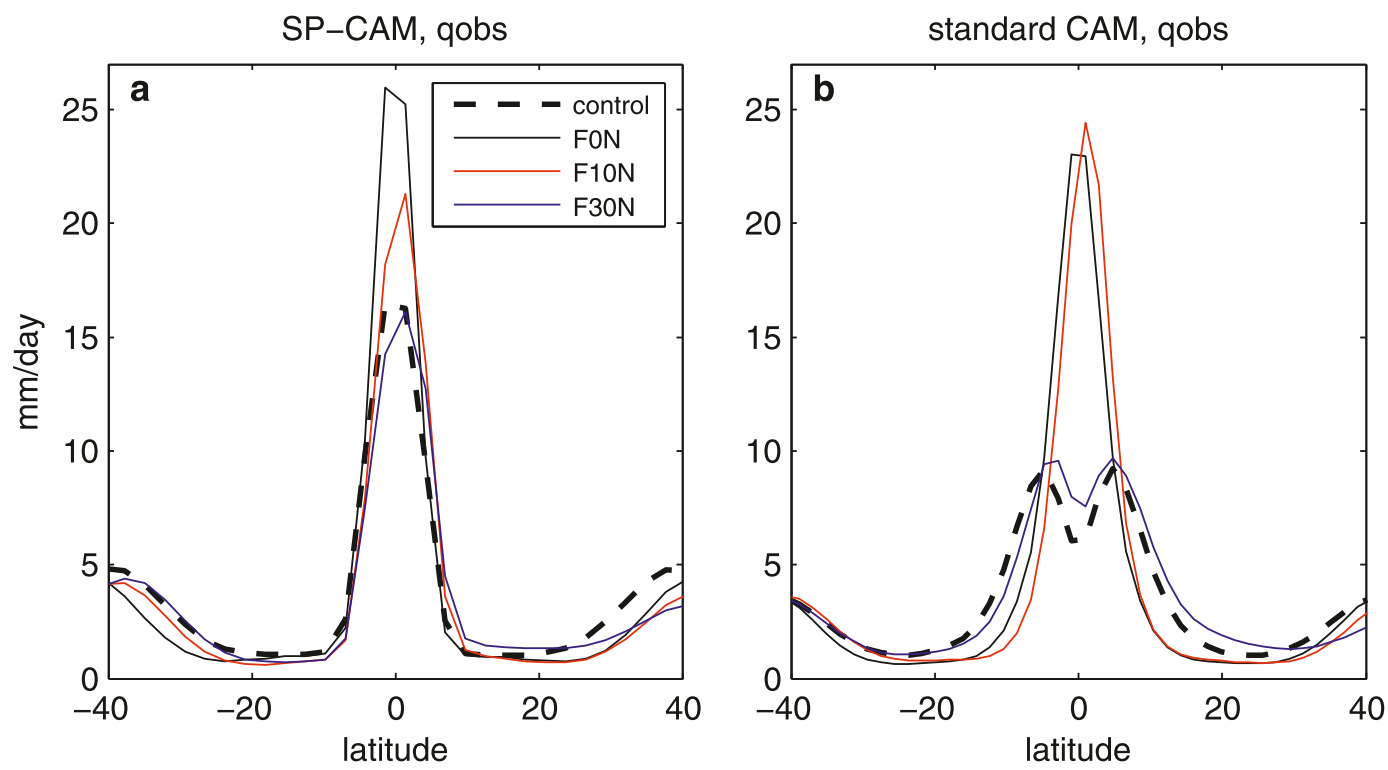

FIG. 12. Zonal-mean precipitation for integrations with a meridional SST gradient in (a) SP-CAM and (b) the standard moist CAM. Dashed black line shows precipitation in the basic state, and solid lines show total precipitation for forcings indicated in the legend.

response in the dry model with a baroclinic basic state presented in SB12 and constitutes a meridional expansion of the basic state's Hadley circulation and a poleward shift of the eddy-driven midlatitude circulation, including the westerly jet. The Northern Hemisphere extratropical EMFC response has a similar spatial structure for all of the torques in the baroclinic basic state but is weaker in amplitude for the $30^{\circ} \mathrm{N}$ torque, consistent with the weaker $\bar{u}$ and $\Psi$ response in the Northern Hemisphere extratropics (e.g., Fig. 13f). Phase speed spectra of the horizontal component of the eddy momentum flux (HEMF, following Randel and Held 1991) confirm that the equatorial torque causes a poleward shift of the HEMF structure together with a poleward shift in the critical line for baroclinic waves (Fig. 16b). ${ }^{4}$ As discussed by SB12 in the context of dry GCMs, this shift of the critical line can be at least qualitatively viewed as being directly forced by the applied torque: $\bar{u}$ becomes more westward in the region of westward forcing as predicted by the linear response. In particular, the poleward HEMF between $20^{\circ}$ and $40^{\circ}$ latitude is well bounded by the critical line in both CAM and SP-CAM; the tropical HEMF (which is much stronger in SP-CAM) is expected to be located on the equatorward side of the critical line since it is associated

\footnotetext{
${ }^{4}$ We switch to showing the flux instead of the flux convergence to more clearly distinguish between the effects of poleward- and equatorward-propagating eddies.
}

with waves propagating away from the equator. SPCAM shows equatorward HEMF on the poleward side of the jet that seems to fall outside the bounds of the critical line, but it is bounded by the envelope of variability of $\bar{u}$ (not shown).

The moist models have a strong positive equatorial EMFC in their basic state that changes in response to the torques. In SP-CAM, all of the torques weaken the equatorial EMFC (Fig. 15). The transient equatorial eddies having the largest variance in the basic state are also weakened by the torque (Figs. 9b,d), indicating that the torque alters tropical EMFC by influencing convectively coupled equatorial waves. Effects on the equatorial wave field were also seen in the barotropic basic state, but the baroclinic basic state possesses a strong convectively coupled Kelvin wave that is clearly shifted to slower eastward phase speeds. Figure 16 confirms that the eddies accomplishing the equatorial HEMF shift to slower eastward phase speeds. The EMFC response in the moist baroclinic basic state thus consists of a tropical response that seems to depend on the details of convectively coupled equatorial waves, and an extratropical response that has much in common with the EMFC response in dry models discussed by SB12.

The tropical EMFC response may help off-equatorial torques produce a meridional shift in the zonal-mean ITCZ (e.g., Figures 12a and 13c show a northward ITCZ shift in response to the $30^{\circ} \mathrm{N}$ torque). As shown in SB12, the dry response to an off-equatorial, westward, upperlevel torque consists of zonal-mean tropical ascent 

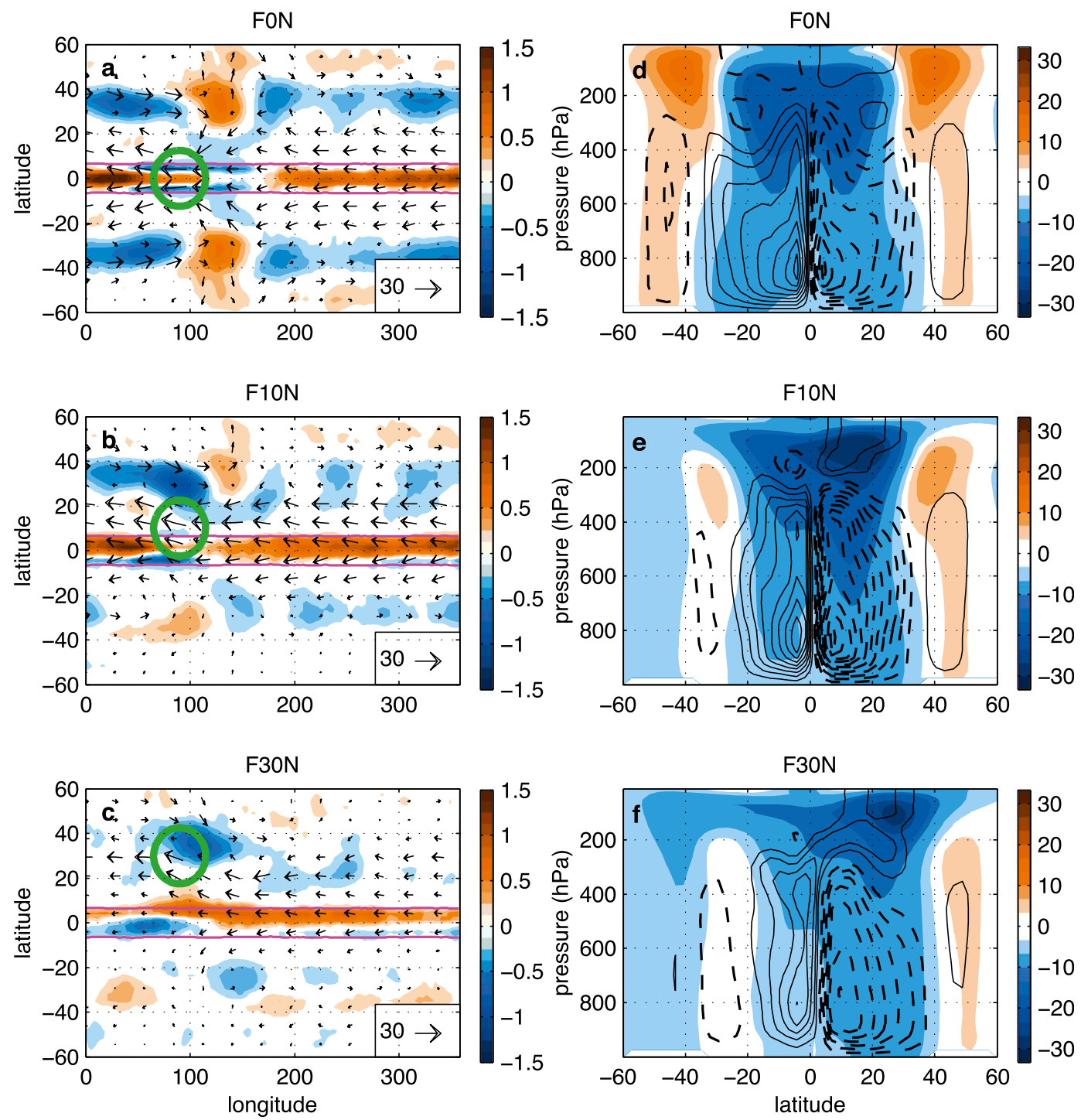

FIG. 13. Response to torques in SP-CAM with a meridional SST gradient. (a)-(c) As in Fig. 5 and (d)-(f) as in Fig. 7.

(left) Magenta lines mark $w=0$ at $500 \mathrm{hPa}$ in the basic state.

(tropical subsidence occurs in some longitudes, but not in the zonal mean). It is thus not obvious how a torque can reduce zonal-mean ascent on or very near the equator, which is necessary to shift the zonal-mean ITCZ. All of the torques in the moist, baroclinic basic state of SPCAM produce a negative equatorial EMFC response with meridional scale smaller than that of the applied torque, and the linear, zonal-mean response to an uppertropospheric, equatorial westward forcing consists of equatorial ascent and off-equatorial subsidence. The $\Psi_{e}$ field shows that the subsidence needed to balance this EMFC response occurs within $10^{\circ}$ of the equator for all of the torques (Fig. 15). A caveat is that the $\Psi_{e}$ field gives the linear response to a specified EMFC, and nonlinear advection by the MMC becomes important close to the equator. In particular, the ratio of the local vertical component of relative vorticity to the Coriolis parameter [as used by Walker and Schneider (2006)] exceeds 0.6 between $0^{\circ}$ and $10^{\circ} \mathrm{S}$ in the upper troposphere for all of the torques (not shown). Because the EMFC response consists of a reduction in the positive basic-state equatorial EMFC, this is equivalent to saying that the torques suppress tropical EMFC and bring the circulation closer to an angular momentum conserving limit, at least in SPCAM's baroclinic basic state.

The extratropical response to the torques has a strong zonally symmetric component that can be explained in terms of a meridional shift in the midlatitude circulation, 


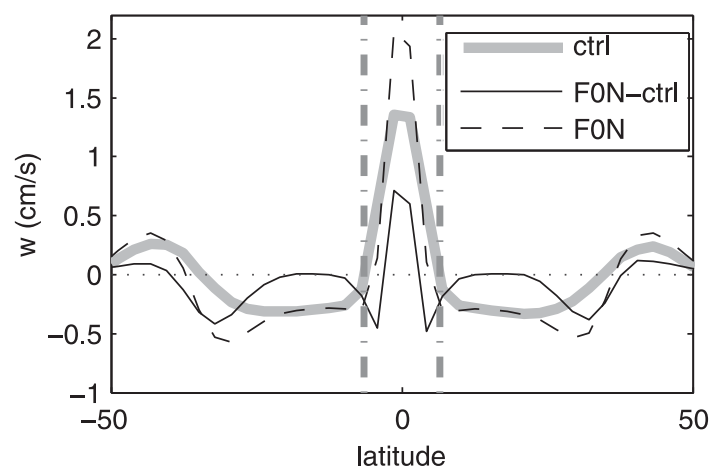

FIG. 14. Limited zonal mean from $40^{\circ}$ to $110^{\circ} \mathrm{E}$ of $500-\mathrm{hPa} w$ in SP-CAM with a meridional SST gradient and the equatorial torque. Gray line is for the basic state, solid black line is the anomaly relative to the basic state, and dashed line is the total vertical velocity in the integration with the forcing. Vertical dasheddotted lines mark the latitudes at which $w=0$ at $500 \mathrm{hPa}$ in the basic state.

as discussed above, but there is also a strong zonal asymmetry in longitudes local to the torque (e.g., near $30^{\circ} \mathrm{N}, 120^{\circ} \mathrm{E}$ in Fig. 13a). Although it is difficult to discern the cause of this asymmetry from the $w$ response, the total upper-level $v$ has a pattern suggestive of a Rossby wave train propagating from the forcing region into the midlatitude waveguide (not shown). Calculation of an approximate refractive index for stationary Rossby waves, roughly following Nishii and Nakamura (2005), confirms that the time-mean patterns of refractive index, $v$, and $w$ are all qualitatively consistent with the hypothesis that the forcing excites a Rossby wave train that propagates along a midlatitude waveguide, with the zonal symmetry of the waveguide broken by the torque.

As in the barotropic basic state, the response to torques in the baroclinic basic state is sensitive to model details. Tropical torques cause the double ITCZ in the standard CAM basic state to merge into a single ITCZ (Fig. 12b). The tropical response in the standard CAM is similarly confined within the basic-state ITCZ, but has a more complicated structure with equatorial ascent east of the torque having two peaks in longitude (not shown). The convectively coupled equatorial wave field is markedly different in the basic states of SP-CAM and the standard $\mathrm{CAM}$, and the response of this wave field and its associated EMFC to torques is also quite different; details are not given here, but this large difference in the equatorial wave field motivated our use of SP-CAM as the primary moist GCM. The extratropical response is also sensitive to model details, with the standard CAM having a weaker shift in the westerly jet and the midlatitude HEMF field (Fig. 16a). While it is unclear how much of the difference between the responses in CAM and SP-CAM is due to a direct sensitivity to model physics and how much is
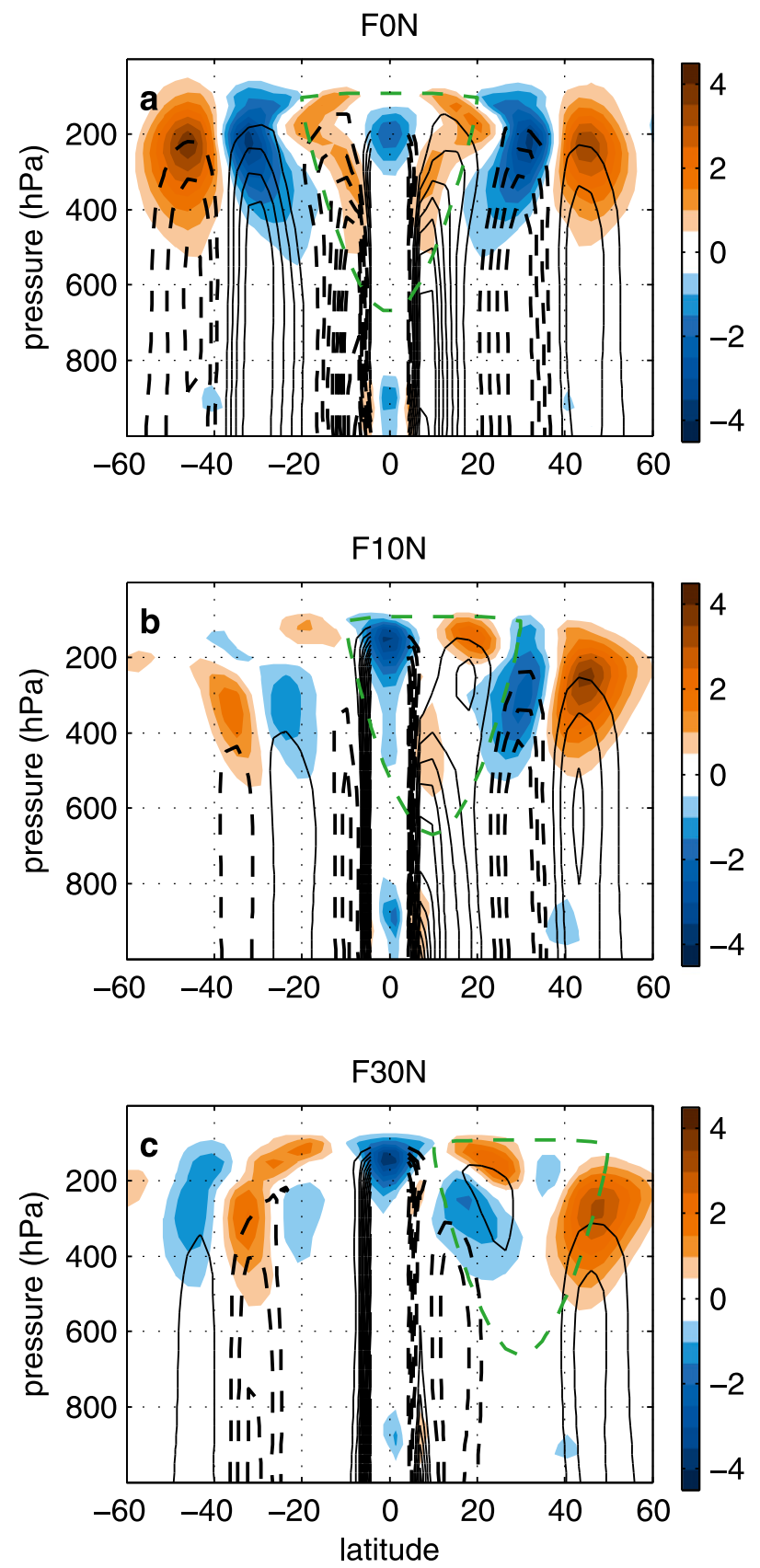

FIG. 15. As in Fig. 8, but for the SP-CAM response with a meridional SST gradient.

due to a sensitivity to the basic state, the important point is that both tropical and midlatitude responses to an imposed torque change with the representation of moist processes.

\section{Summary and discussion}

This study examined the tropospheric response to prescribed momentum forcings in several idealized moist 

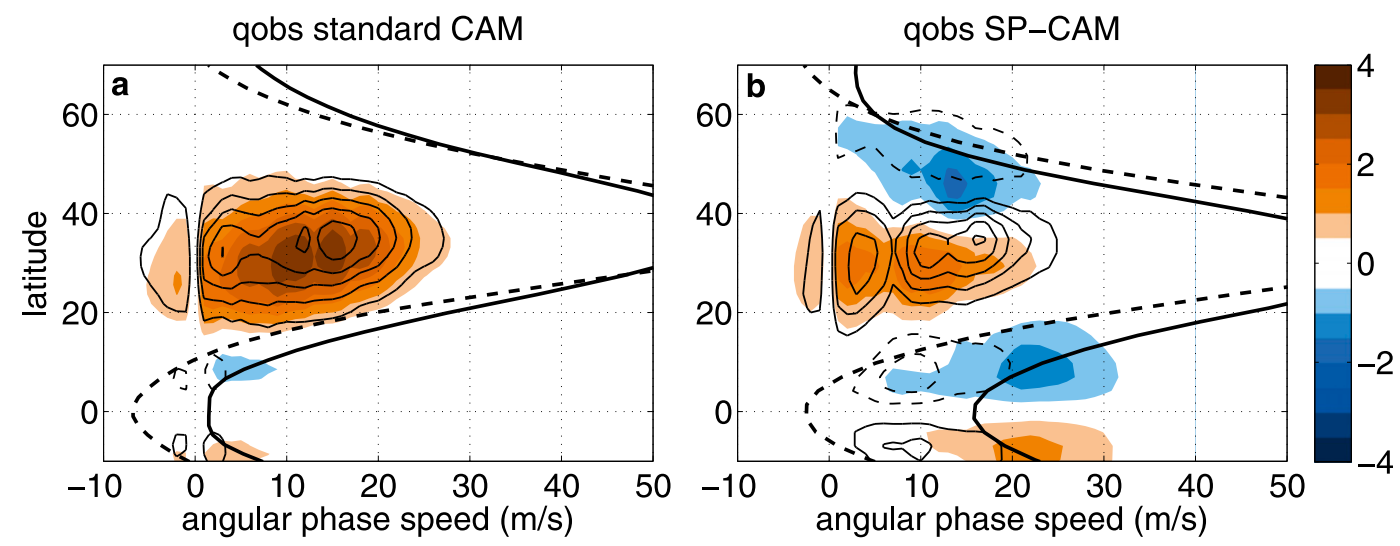

FIG. 16. Phase speed spectra vs latitude of the HEMF at $200 \mathrm{hPa}$ for (a) the standard CAM and (b) SP-CAM with a meridional SST gradient. Colors show HEMF in the basic state and contours (negative dashed) show total HEMF in runs with the equatorial torque. Contour interval is $0.5 \mathrm{~m}^{2} \mathrm{~s}^{-2}$. The total zonal-mean zonal wind at $200 \mathrm{hPa}$ is shown for the basic state (thick solid line) and for integrations with the equatorial torque (thick dashed line).

models. The goal was to improve understanding of circulations induced by zonally confined torques, such as those produced by OGWD and CMT. Zonally confined, westward, upper-tropospheric torques were shown by SB12 to produce a tropical response in dry models that is qualitatively described by the linear dynamics of equatorially trapped waves, with equatorial ascent east of tropical torques and off-equatorial subsidence to the west. Here we showed that the diabatic effects of moist convection amplify the vertical motion response to a torque by reducing the effective static stability. This amplification can only occur in precipitating regions, and the amplitude of subsidence saturates when clear-sky radiative cooling balances the total adiabatic warming produced by subsidence. For basic states in which precipitation is concentrated in an intertropical convergence zone (ITCZ), these effects cause much of the vertical motion response to be confined to the basic-state ITCZ, even when the torque is remote from the ITCZ.

Nonlinear advection of dynamic and thermodynamic quantities is shown to alter the response to a torque, both in steady solutions of damped models like the QTCM and in time-mean solutions of GCMs with nearly inviscid free tropospheres. In particular, meridional momentum advection by eddies is associated with secondary meridional overturning circulations that modify the zonal-mean response to a torque. These eddies include transient, convectively coupled tropical disturbances as well as, in a baroclinic basic state, extratropical baroclinic waves. The former are highly sensitive to model details such as representations of moist convection, and the zonal-mean response to a torque is similarly sensitive. In contrast, perturbations to the extratropical wave field are qualitatively described by the dry dynamics discussed by SB12, with westward tropical torques typically producing a poleward shift in both the critical surface for baroclinic eddies and the entire midlatitude circulation.

One practical result is that both tropical and subtropical torques can produce meridional shifts in the ITCZ. Previous studies have shown that tropical CMT can alter the ITCZ (Richter and Rasch 2008; Wu et al. 2003), and our results provide additional dynamical insight into the effects seen in those studies. Our results also show that this can be a highly nonlocal response, with a torque centered at $30^{\circ} \mathrm{N}$ shifting an equatorial ITCZ and perturbing the Southern Hemisphere Hadley and Ferrel cells. This may be of particular relevance for Asian OGWD, which has zonal-mean amplitude and meridional location roughly similar to that of our $30^{\circ} \mathrm{N}$ torque (e.g., McFarlane 1987). Such subtropical torques do not project strongly on equatorial wave modes, but instead produce a zonally confined form of a downwardcontrol response (Haynes et al. 1991), with subsidence poleward of the torque, ascent equatorward, and local poleward flow producing a Coriolis force that balances the westward forcing. Although the gross moist stability does not directly influence the total mass flux in the zonal-mean meridional overturning, moist convection does regionally amplify the vertical motion response to such subtropical torques in our models (e.g., Figs. 5c,f). These idealized results suggest that Asian OGWD might perturb the tropical ITCZ and suppress rainfall over northern Asia. Of course, Himalayan OGWD is largest in boreal winter when the ITCZ is located south of the equator, and our basic-state ITCZ was always centered on the equator. Further studies using models with more realistic basic states and boundary conditions are needed to determine how the dynamics are modified by such 
complications. Previous investigations have integrated GCMs with and without parameterized OGWD in the presence of realistic boundary conditions and forcings, but a focused, mechanistic examination of the tropical precipitation response to OGWD still seems lacking. Shin et al. (2010) found little change in boreal winter precipitation when parameterized OGWD was turned off in a global version of the Weather Research and Forecasting model (WRF), but Slingo and Pearson (1987) found OGWD suppressed boreal winter Eurasian rainfall. Other studies (Zhou et al. 1996; Kirtman et al. 1993) found a substantial tropical precipitation response to OGWD, albeit over periods of a single season or less.

The strong influence of tropical and subtropical torques on the equatorial wave field and its associated EMFC highlights the importance of properly representing convectively coupled equatorial waves if one wishes to properly represent the effects of atmospheric momentum transfers, even by processes such as OGWD that are not typically thought to be closely coupled with the equatorial wave field. Tropical and subtropical torques in general produced large modifications of the ITCZ and the equatorial wave field in both SP-CAM and the standard CAM, and the character of this modification differed greatly between the two models. However, we did not clearly separate the sensitivity of the response to model formulation from the sensitivity to the basic state. Further work with idealized models may help in this task. For example, Dias and Pauluis (2009) showed that, in a theoretical model, the effect of moist convection on free Kelvin and Rossby waves depends on the meridional width of the basic-state ITCZ. Their theoretical model could be used to better understand the effect of mechanically forced waves propagating along an ITCZ.

Another notable result is that tropical torques can merge a double ITCZ into a single ITCZ. The idea that CMT might influence the ITCZ in this way was suggested by Khairoutdinov et al. (2005) based on two integrations of SP-CAM, conducted with and without CMT, both with realistic boundary conditions. Our results may provide a simple way of understanding this effect. The vertical structure of CMT may have more in common with our purely baroclinic forcings than with forcings trapped at upper levels, since it can have high amplitude in the lower troposphere owing to transports by shallow convection (e.g., Carr and Bretherton 2001; Lin et al. 2005). Although GCM parameterizations of CMT produce a vertical structure that is more complicated than that of our purely baroclinic forcing, a zonal-mean parameterized CMT eastward tendency of $1 \mathrm{~m} \mathrm{~s}^{-1}$ day $^{-1}$ is typical in the tropical lower troposphere, with a westward tendency in the mid- to upper troposphere (e.g., Gregory et al. 1997; Song et al. 2008). Results from our purely baroclinic QTCM and the dry models of SB12 show that such a tropical forcing would produce a zonal-mean linear response consisting of equatorial ascent and off-equatorial subsidence-precisely what is needed to merge a double ITCZ into a single equatorial ITCZ. Song et al. (2008) discussed similar dynamics, albeit in a model in which moist convection did not interact with large-scale flow.

The forcings used in this study did not interact with the flow, and so have questionable realism. For example, the zonal acceleration due to OGWD should have a sign opposite to that of the local surface wind and should depend on the location of the zero-wind line, which acts as a critical layer; some of our forcings clearly violated these constraints. Similarly, CMT interacts with convectively coupled circulations and can produce up-gradient and down-gradient momentum transfers (e.g., Majda and Stechmann 2008, 2009), which could be parameterized but were neglected here. Our results may nevertheless serve as a building block for understanding the influence of OGWD and CMT on large-scale flow. Our results complement studies of the linear response to prescribed thermal forcings (e.g., Gill 1980) and studies of the nonlinear response to zonally symmetric momentum forcings (Ring and Plumb 2007; Chen and Zurita-Gotor 2008), and contribute to a better understanding of wave-mean flow interaction in the atmosphere.

Acknowledgments. This work was supported in part by the facilities and staff of the Yale University Faculty of Arts and Sciences High Performance Computing Center and by the National Science Foundation under Grant CNS 08-21132 that partially funded acquisition of the facilities. W.R.B. acknowledges financial support from Office of Naval Research Young Investigator Program Award N00014-11-1-0617. TAS acknowledges support from the David and Lucile Packard Foundation. We thank Brian Dobbins for help with model configuration. Two anonymous reviewers provided constructive comments that improved the manuscript.

\section{APPENDIX}

\section{Zonal-Mean Mass Streamfunction in Mechanically Forced Viscous Models}

It was shown in the body of this paper that coupling with moist convection increased the peak amplitude of the zonal-mean mass streamfunction in the QTCM but not in the GCMs. This appendix explains why mechanical damping through the depth of the troposphere makes the zonal-mean overturning directly sensitive to the static stability and the gross moist stability. 
The QTCM is a highly damped model, compared to GCMs, as seen by a steady, linear, $\beta$-plane version of the zonal-mean QTCM equations:

$$
\begin{aligned}
-\beta y \bar{v} & =\overline{\mathcal{F}}-\epsilon_{u} \bar{u}, \\
\beta y \bar{u} & =-\kappa \frac{\partial \bar{T}}{\partial y}-\epsilon_{v} \bar{v}, \quad \text { and } \\
\left(M_{s}-M_{q}\right) \frac{\partial \bar{v}}{\partial y} & =\frac{g}{p_{T}} \bar{Q}^{\text {net }},
\end{aligned}
$$

where overbars represent zonal means, $\epsilon_{u}$ and $\epsilon_{v}$ are coefficients of linear damping, $\kappa$ is the ratio of the gas constant to the specific heat of air at constant pressure, and $p_{T}$ is the pressure depth of the troposphere. This is a steady, linear form of (5.1)-(5.5) from Neelin and Zeng (2000), adapted to an equatorial $\beta$ plane. Variables represent vertically integrated properties of the first baroclinic mode of the QTCM. The last equation is a moist static energy equation, obtained by combining the temperature and moisture equations, with $Q^{\text {net }}$ the net flux of moist static energy into the tropospheric column. This shows that $\sqrt{M_{s}}$ is the dry gravity wave speed and $\sqrt{M_{s}-M_{q}}$ is the moist convectively coupled gravity wave speed.

If $\mathcal{F} \neq 0$, a mechanically forced meridional overturning will exist that can be represented simply if $Q^{\text {net }}$ consists only of linear damping on the same time scale used to damp momentum (i.e., $1 / \epsilon_{u}$ ). In that case, with the additional assumption that $\epsilon_{v} \bar{v}$ can be neglected in (A2) as in Gill (1980), (A1)-(A3) can be simplified to a parabolic cylinder equation:

$$
\frac{\partial^{2} \bar{v}}{\partial y^{2}}-\frac{\beta^{2}}{\kappa\left(M_{s}-M_{q}\right)} y^{2} \bar{v}=\frac{\beta}{\kappa\left(M_{s}-M_{q}\right)} y \overline{\mathcal{F}} .
$$

This can be nondimensionalized using the length scale

$$
L_{0}=\left(\frac{\kappa M_{s}}{4 \beta^{2}}\right)^{1 / 4},
$$

the velocity scale $\sqrt{M_{s}}$, and the mechanical forcing scale $\sqrt{\kappa} M_{s} / L_{0}$ to obtain

$$
\frac{\partial^{2} \bar{v}}{\partial y^{2}}-\frac{1}{4 c_{0}^{2}} y^{2} \bar{v}=\frac{1}{2 c_{0}^{2}} y \overline{\mathcal{F}},
$$

where the nondimensional moist gravity wave speed is

$$
c_{0}^{2}=\frac{M_{s}-M_{q}}{M_{s}} .
$$

Expression (A6) is identical to the nondimensional moist system studied by Dias and Pauluis (2009), but with a mechanical instead of a thermal forcing. It is also equivalent to (1) from SB12 but with damping eliminated by use of the long-wave approximation in (A2) and with the introduction of the moist gravity wave speed $c_{0}$. Solutions to the homogeneous version of (A6) can be written in terms of parabolic cylinder functions:

$$
\bar{v}(y)=D_{-1 / 2}\left(y / \sqrt{c_{0}}\right),
$$

so that free solutions are reduced in meridional scale by a factor of $\sqrt{c_{0}}$. To find analytical solutions for the dry case of $c_{0}=1$, SB12 expressed $\overline{\mathcal{F}}$ as the first parabolic cylinder function $D_{1}(y)$, allowing solutions to be found in terms of $D_{1}(y)$. But when $c_{0} \neq 1$, the same forcing $D_{1}(y)$ must be expanded in terms of higher order $D_{n}$ in the rescaled coordinate $y / \sqrt{c_{0}}$, and the solution must be found in terms of a series of odd $D_{n}$ in the rescaled coordinate. Instead of finding this analytical series solution, we present numerical solutions from the QTCM for multiple values of $c_{0}$, which show that as $c_{0}$ decreases, the amplitude of $\bar{v}$ increases and its meridional scale is slightly reduced (Fig. 2c).

Several aspects of the zonal-mean QTCM response to a torque can be understood using (A6), which confirms that $\bar{v}$ depends on the gross moist stability but is independent of the damping, at least under the long-wave approximation and the idealization that momentum and moist static energy anomalies are damped on similar time scales. Very near the equator, $\overline{\mathcal{F}}$ is balanced by the first term on the left-hand side of (A6) so that the curvature of $\bar{v}$ must vary inversely with $c_{0}$. Physically, the torque is balanced by drag on $u$, which in turn is in thermal wind balance with the meridional temperature gradient created adiabatically by the meridional circulation. As the stability (i.e., $c_{0}$ ) increases, a weaker meridional circulation can produce the temperature gradient and thus the drag needed to balance the torque. The sensitivity of the zonal-mean meridional circulation to the effective static stability is thus a characteristic of models that damp $u$ throughout the troposphere. These dynamics are consistent with the discussion by Song et al. (2008) of the anomalous Hadley circulation forced by a CMT tendency on $\bar{u}$ in their GCM.

\section{REFERENCES}

Boos, W. R., and K. A. Emanuel, 2008: Wind-evaporation feedback and the axisymmetric transition to angular momentum conserving Hadley flow. J. Atmos. Sci., 65, 3758-3778.

Carr, M. T., and C. S. Bretherton, 2001: Convective momentum transport over the tropical pacific: Budget estimates. J. Atmos. Sci., 58, 1673-1693. 
Chen, G., and P. Zurita-Gotor, 2008: The tropospheric jet response to prescribed zonal forcing in an idealized atmospheric model. J. Atmos. Sci., 65, 2254-2271.

Collins, W., and Coauthors, 2006: The formulation and atmospheric simulation of the Community Atmosphere Model version 3 (CAM3). J. Climate, 19, 2144-2161.

DeMott, C., D. Randall, and M. Khairoutdinov, 2007: Convective precipitation variability as a tool for general circulation model analysis. J. Climate, 20, 91-112.

Dias, J., and O. Pauluis, 2009: Convectively coupled waves propagating along an equatorial ITCZ. J. Atmos. Sci., 66, 22372255.

Emanuel, K. A., J. D. Neelin, and C. S. Bretherton, 1994: On largescale circulations in convecting atmospheres. Quart. J. Roy. Meteor. Soc., 120, 1111-1143.

Frierson, D., 2007: Convectively coupled kelvin waves in an idealized moist general circulation model. J. Atmos. Sci., 64, 2076-2090.

Fritts, D. C., and M. J. Alexander, 2003: Gravity wave dynamics and effects in the middle atmosphere. Rev. Geophys., 41,1003, doi:10.1029/2001RG000106.

Gill, A. E., 1980: Some simple solutions for heat-induced tropical circulation. Quart. J. Roy. Meteor. Soc., 106, 447-462.

Gregory, D., R. Kershaw, and P. M. Inness, 1997: Parameterization of momentum transport by convection. II: Tests in single column and general circulation models. Quart. J. Roy. Meteor. Soc., 123, 1153-1183.

Hack, J. J., 1994: Parameterization of moist convection in the National Center for Atmospheric Research Community Climate Model (CCM2). J. Geophys. Res., 99 (D3), 55515568.

Haynes, P. H., C. J. Marks, M. E. McIntyre, and T. G. Shepherd, 1991: On the "downward control" of extratropical diabatic circulations by eddy-induced mean zonal forces. J. Atmos. Sci., 48, 651-678.

Held, I. M., and M. Suarez, 1994: A proposal for the intercomparison of the dynamical cores of atmospheric general circulation models. Bull. Amer. Meteor. Soc., 75, 1825-1830.

Khairoutdinov, M., and D. Randall, 2001: A cloud resolving model as a cloud parameterization in the NCAR Community Climate System Model: Preliminary results. Geophys. Res. Lett., 28, 3617-3620.

,-- , and C. Demott, 2005: Simulations of the atmospheric general circulation using a cloud-resolving model as a superparameterization of physical processes. J. Atmos. Sci., 62, 2136-2154.

Kiladis, G., M. Wheeler, P. Haertel, K. Straub, and P. Roundy, 2009: Convectively coupled equatorial waves. Rev. Geophys., 47, RG2003, doi:10.1029/2008RG000266.

Kirtman, B., A. Vernekar, D. DeWitt, and J. Zhou, 1993: Impact of orographic gravity wave drag on extended-range forecasts with the COLA-GCM. Atmósfera, 6, 3-23.

Lin, J.-L., M. Zhang, and B. Mapes, 2005: Zonal momentum budget of the Madden-Julian oscillation: The source and strength of equivalent linear damping. J. Atmos. Sci., 62, 2172-2188.

Majda, A., and S. Stechmann, 2008: Stochastic models for convective momentum transport. Proc. Natl. Acad. Sci. USA, 105, $17614-17619$

- and - 2009: The skeleton of tropical intraseasonal oscillations. Proc. Natl. Acad. Sci. USA, 106, 8417-8422.

Matsuno, T., 1966: Quasi-geostrophic motions in the equatorial area. J. Meteor. Soc. Japan, 44, 25-43.
McFarlane, N. A., 1987: The effect of orographically excited gravity-wave drag on the circulation of the lower stratosphere and troposphere. J. Atmos. Sci., 44, 1775-1800.

Neale, R. B., and B. J. Hoskins, 2001: A standard test for AGCMs including their physical parameterizations. I: The proposal. Atmos. Sci. Lett., 1, 101-107.

- and Coauthors, 2010: Description of the NCAR Community Atmosphere Model (CAM 5.0). NCAR Tech. Note NCAR/ TN-486+STR, 282 pp.

Neelin, J. D., and I. M. Held, 1987: Modeling tropical convergence based on the moist static energy budget. Mon. Wea. Rev., 115, 3-12.

_ , and N. Zeng, 2000: A quasi-equilibrium tropical circulation model: Formulation. J. Atmos. Sci., 57, 1741-1766.

Nishii, K., and H. Nakamura, 2005: Upward and downward injection of Rossby wave activity across the tropopause: A new aspect of the troposphere-stratosphere dynamical linkage. Quart. J. Roy. Meteor. Soc., 131, 545-564.

Norton, W. A., 2006: Tropical wave driving of the annual cycle in tropical tropopause temperatures. Part II: Model results. J. Atmos. Sci., 63, 1420-1431.

Palmer, T. N., G. J. Schutts, and R. Swinbank, 1986: Alleviation of a systematic westerly bias in general circulation and numerical weather prediction models through an orographic gravity wave drag parameterization. Quart. J. Roy. Meteor. Soc., 112, 1001-1039.

Randel, W. J., and I. M. Held, 1991: Phase speed spectra of transient eddy fluxes and critical layer absorption. J. Atmos. Sci., 48, 688-697.

Richter, J. H., and P. J. Rasch, 2008: Effects of convective momentum transport on the atmospheric circulation in the Community Atmosphere Model, Version 3. J. Climate, 21, 1487-1499.

Ring, M. J., and R. A. Plumb, 2007: Forced annular mode patterns in a simple atmospheric general circulation model. J. Atmos. Sci., 64, 3611-3626.

Seager, R., R. Murtugudde, A. Clement, and C. Herweijer, 2003: Why is there an evaporation minimum at the equator? $\mathrm{J}$. Climate, 16, 3793-3802.

Shaw, T., and W. Boos, 2012: The tropospheric response to tropical and subtropical zonally asymmetric torques: Analytical and idealized numerical model results. J. Atmos. Sci., 69, 214-235.

Shin, H. H., S.-Y. Hong, J. Dudhia, and Y.-J. Kim, 2010: Orographyinduced gravity wave drag parameterization in the global WRF: Implementation and sensitivity to shortwave radiation schemes. Adv. Meteor., 2010, 959014, doi:10.1155/ 2010/959014.

Slingo, A., and D. Pearson, 1987: A comparison of the impact of an envelope orography and of a parametrization of orographic gravity-wave drag on model simulations. Quart. J. Roy. Meteor. Soc., 113, 847-870.

Sobel, A., and C. Bretherton, 2000: Modeling tropical precipitation in a single column. J. Climate, 13, 4378-4392.

Song, X., X. Wu, G. J. Zhang, and R. W. Arritt, 2008: Dynamical effects of convective momentum transports on global climate simulations. J. Climate, 21, 180-194.

Stephenson, D. B., 1994: The northern hemisphere tropospheric response to changes in the gravity-wave drag scheme in a perpetual January GCM. Quart. J. Roy. Meteor. Soc., 120, 699-712.

Stevens, D. E., 1979: Vorticity, momentum and divergence budgets of synoptic-scale wave disturbances in the tropical eastern Atlantic. Mon. Wea. Rev., 107, 535-550. 
Suarez, M., and D. Duffy, 1992: Terrestrial superrotation: A bifurcation of the general circulation. J. Atmos. Sci., 49, 15411554.

Walker, C. C., and T. Schneider, 2006: Eddy influences on Hadley circulations: Simulations with an idealized GCM. J. Atmos. Sci., 63, 3333-3350.

Wheeler, M., and G. N. Kiladis, 1999: Convectively coupled equatorial waves: Analysis of clouds and temperature in the wavenumber-frequency domain. J. Atmos. Sci., 56, 374-399.

Wu, X., X. Liang, and G. Zhang, 2003: Seasonal migration of ITCZ precipitation across the equator: Why can't GCMs simulate it? Geophys. Res. Lett., 30, 1824, doi:10.1029/ 2003GL017198.

Yu, J., C. Chou, and J. Neelin, 1998: Estimating the gross moist stability of the tropical atmosphere. J. Atmos. Sci., 55, 1354-1372.

Zhang, G., and N. McFarlane, 1995: Sensitivity of climate simulations to the parameterization of cumulus convection in the Canadian Climate Centre general circulation model. Atmos.Ocean, 33, 407-407.

Zhou, J., Y. Sud, and K. Lau, 1996: Impact of orographically induced gravity-wave drag in the GLA GCM. Quart. J. Roy. Meteor. Soc., 122, 903-927. 